Supplement of The Cryosphere, 15, 1663-1675, 2021

https://doi.org/10.5194/tc-15-1663-2021-supplement

(C) Author(s) 2021. CC BY 4.0 License.

(c) (1)

Supplement of

\title{
Calving Front Machine (CALFIN): glacial termini dataset and automated deep learning extraction method for Greenland, 1972-2019
}

Daniel Cheng et al.

Correspondence to: Daniel Cheng (dlcheng@uci.edu)

The copyright of individual parts of the supplement might differ from the article licence. 


\section{Temporal Availability}

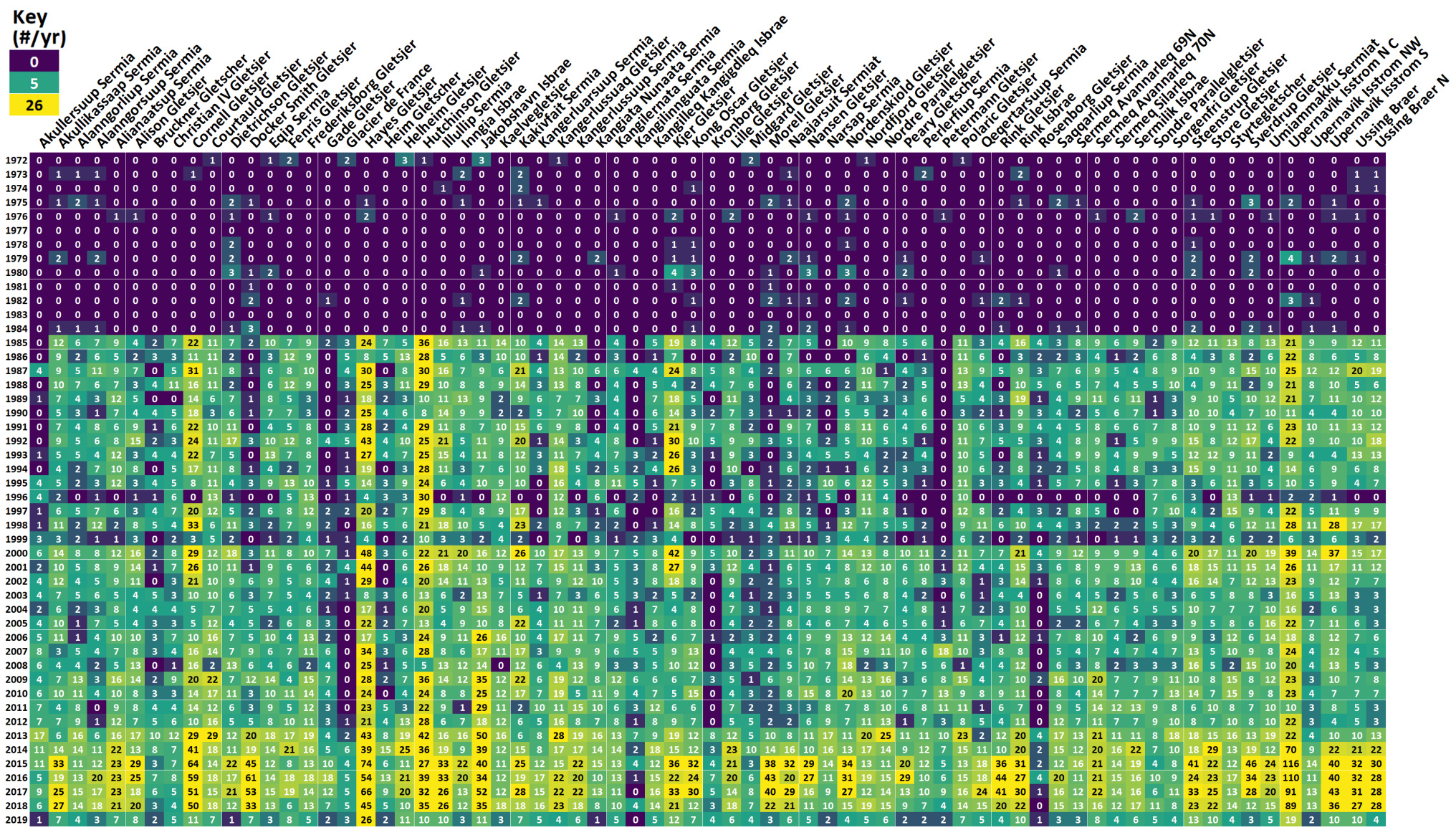

Figure S1. Temporal Availability Chart. Listed are the number of fronts per year, for all 66 glaciers in the CALFIN dataset. 


\section{Inter-model Comparison Table}

Table S1. Consolidated validation set and inter-model error metrics.

\begin{tabular}{cccccc}
\hline Validation Set & Model & Mean Distance & Median Distance & IoU Coastline & IoU Ice/Ocean \\
\hline \hline CALFIN-VS & CALFIN-NN & $2.25 \mathrm{px}, 86.76 \mathrm{~m}$ & $1.21 \mathrm{px}, 44.59 \mathrm{~m}$ & 0.4884 & 0.9793 \\
\hline CALFIN-VS-L7-none & CALFIN-NN & $2.27 \mathrm{px}, 81.65 \mathrm{~m}$ & $1.16 \mathrm{px}, 44.01 \mathrm{~m}$ & 0.4880 & 0.9819 \\
CALFIN-VS-L7-only & CALFIN-NN & $2.22 \mathrm{px}, 91.93 \mathrm{~m}$ & $1.33 \mathrm{px}, 49.24 \mathrm{~m}$ & 0.4888 & 0.9766 \\
\hline M-VS & CALFIN-NN & $2.56 \mathrm{px}, 97.72 \mathrm{~m}$ & $2.55 \mathrm{px}, 97.44 \mathrm{~m}$ & 0.3332 & N/A \\
M-VS & M-NN & $1.97 \mathrm{px}, 96.31 \mathrm{~m}$ & N/A & N/A & N/A \\
\hline Z-VS & CALFIN-NN & $2.11 \mathrm{px}, 115.24 \mathrm{~m}$ & $1.65 \mathrm{px}, 77.29 \mathrm{~m}$ & 0.3832 & 0.9761 \\
Z-VS & Z-NN & $17.3 \mathrm{px}, 104 \mathrm{~m}$ & N/A & N/A & N/A \\
\hline B-VS & CALFIN-NN & $2.35 \mathrm{px}, 330.63 \mathrm{~m}$ & $0.74 \mathrm{px}, 112.75 \mathrm{~m}$ & 0.6451 & 0.9879 \\
B-VS & B-NN & $2.69 \mathrm{px}, 108 \mathrm{~m}$ & N/A & N/A & 0.905 \\
\hline
\end{tabular}

\section{Dataset Metadata}

Level 0 products consist of fjord boundary GeoTiff masks, the domain Shapefiles used for subsetting, and the Landsat scene name list text files. Level 1 product consists of a LineString Shapefile with 22678 features, and a Polygon Shapefile with 17771 features, grouped by glacial basin. Both

5 Shapefiles share a feature schema derived from the MEaSUREs glacial terminus positions dataset (NSIDC-0642), as detailed in Table S2.

- Temporal resolution: sub-seasonal

- Spatial resolution: $\sim 30$ meters

- Spatial accuracy: $<90$ meters

- Projection: EPSG:3413 (WGS 84 / NSIDC Sea Ice Polar Stereographic North) 
Table S2. Shapefile Feature Schema Attribute Table

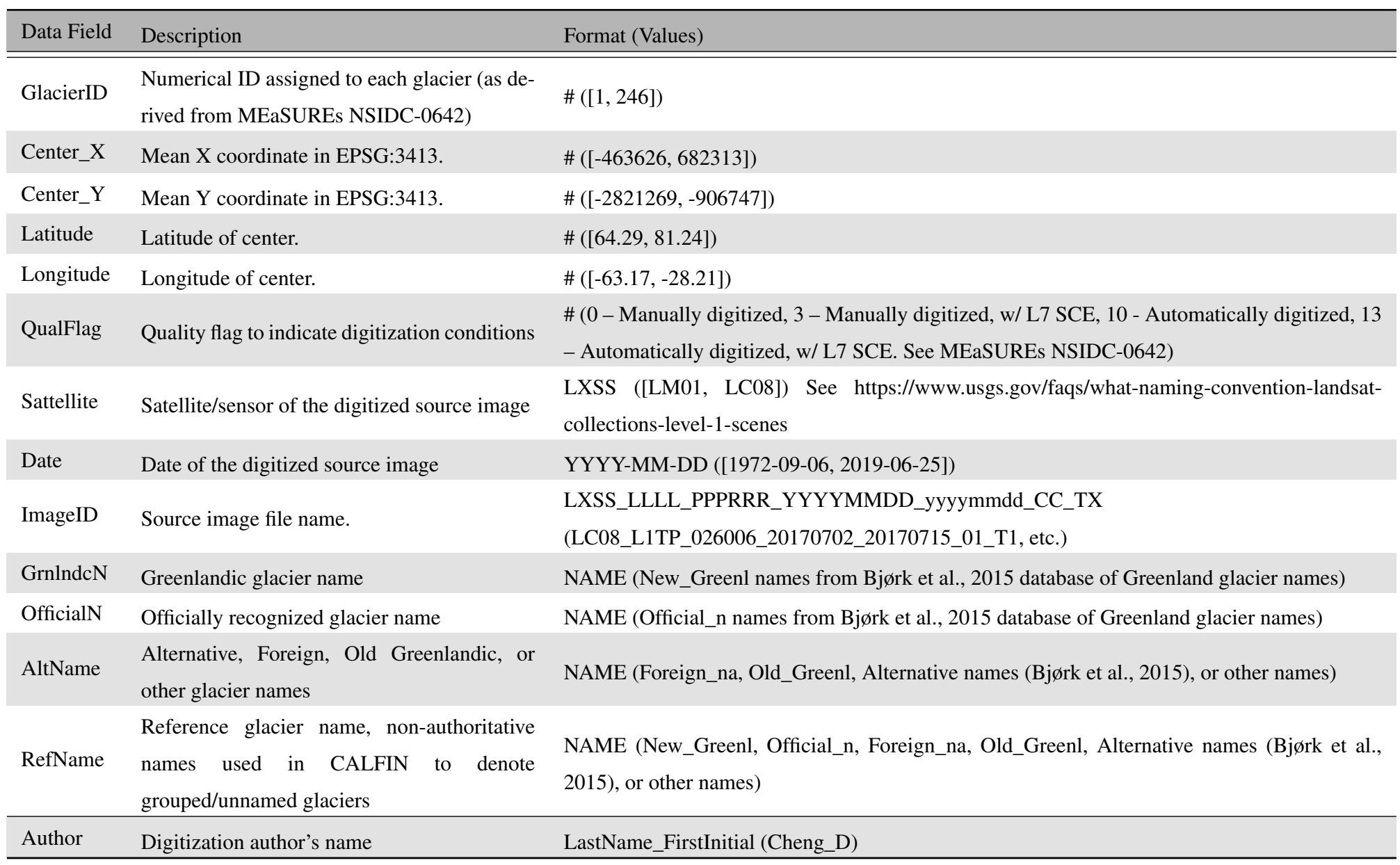




\section{Error Estimation}

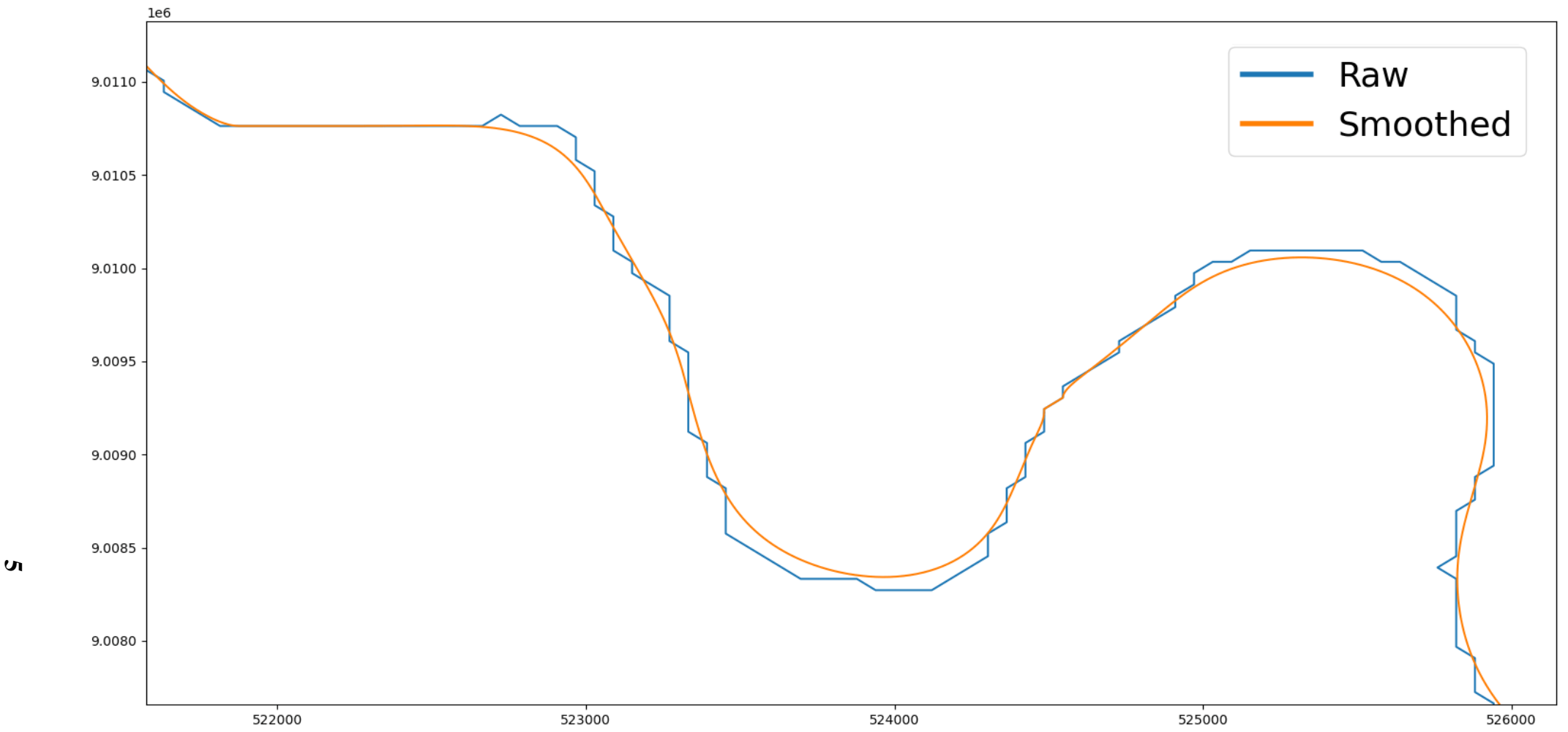

Figure S2. Smoothed versus Raw Polyline The post-processing line smoothing operation turns the raw coastline (blue) into a smoothed data product polyline (orange), deviating by no more than 1 pixel from the raw coastline. Since the variations are on the sub-pixel scale, the error introduced is no more than the uncertainty of the base resolution, and well within the neural network uncertainty. 


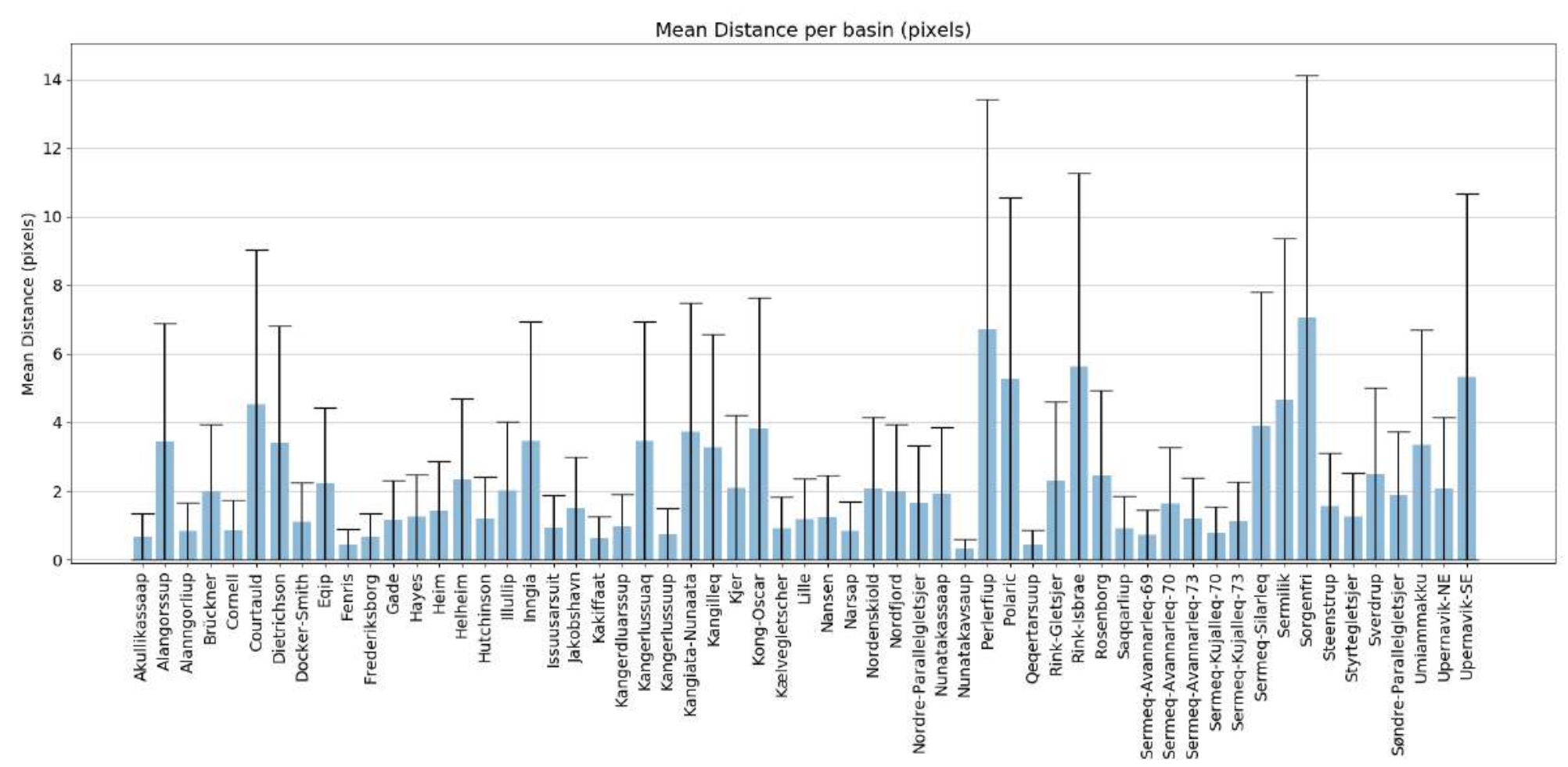

Figure S3. True mean distance error estimates per basin, in pixels. We estimate the true mean distance error per basin to lie between the above intervals with $95 \%$ confidence.

\section{Training Data}



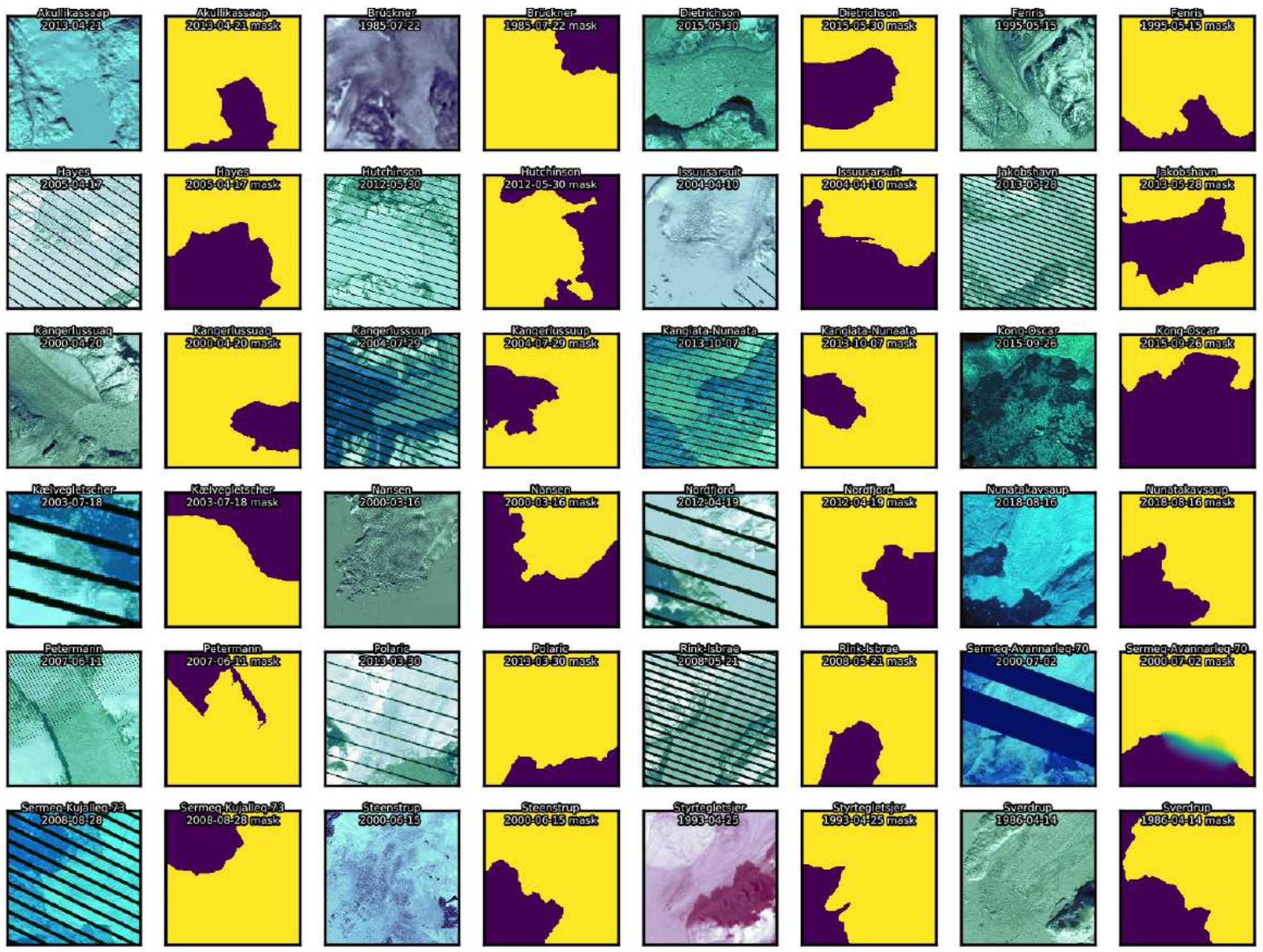

Figure S4. CALFIN-NN training data. Show above is a subset of the 1872 CALFIN-NN training image/mask pairs. Not shown are the coastline masks, which are dynamically generated after performing data augmentation on the above masks, using Canny edge filters and dilation to a 3 pixel wide edge. Note blurred mask in uncertain areas, as in Sermeq Avannarleq 70 2000-07-02. Images are masked at a minimum resolution of 1024 pixels. 


\section{Validation Data}

\subsection{CALFIN Validation Set}

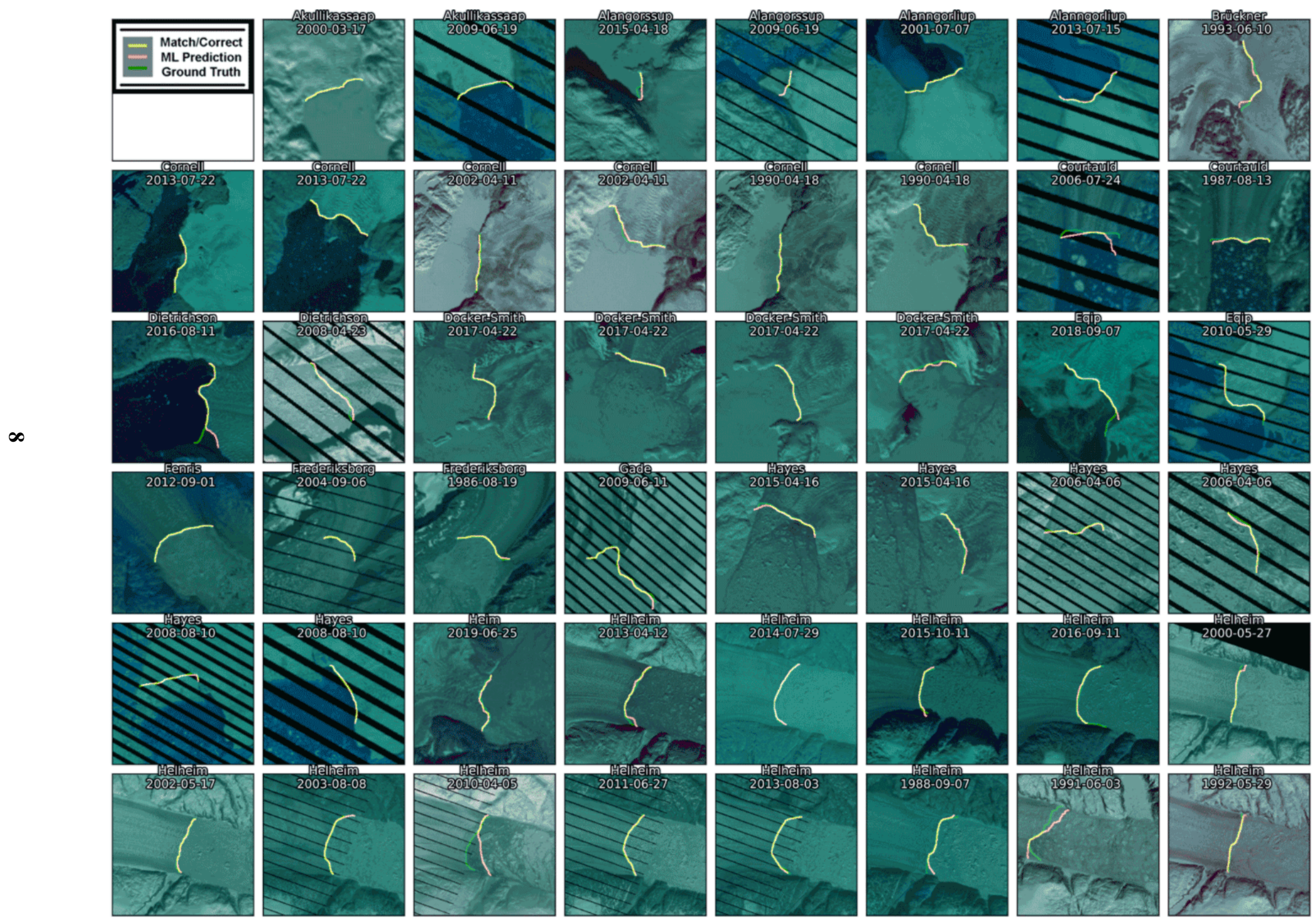

Figure S5. CALFIN validation outputs, part 1. 


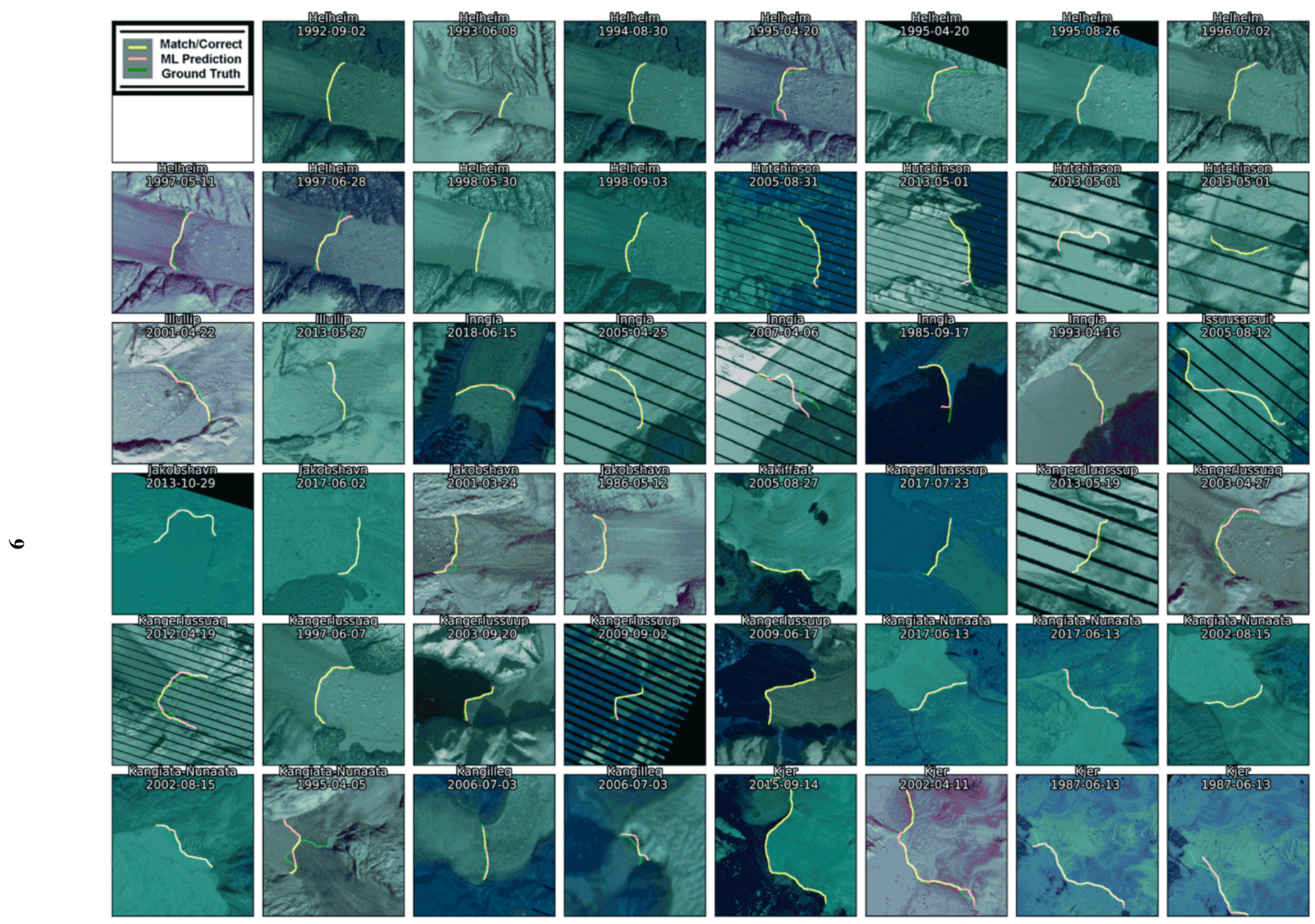

Figure S6. CALFIN validation outputs, part 2. 


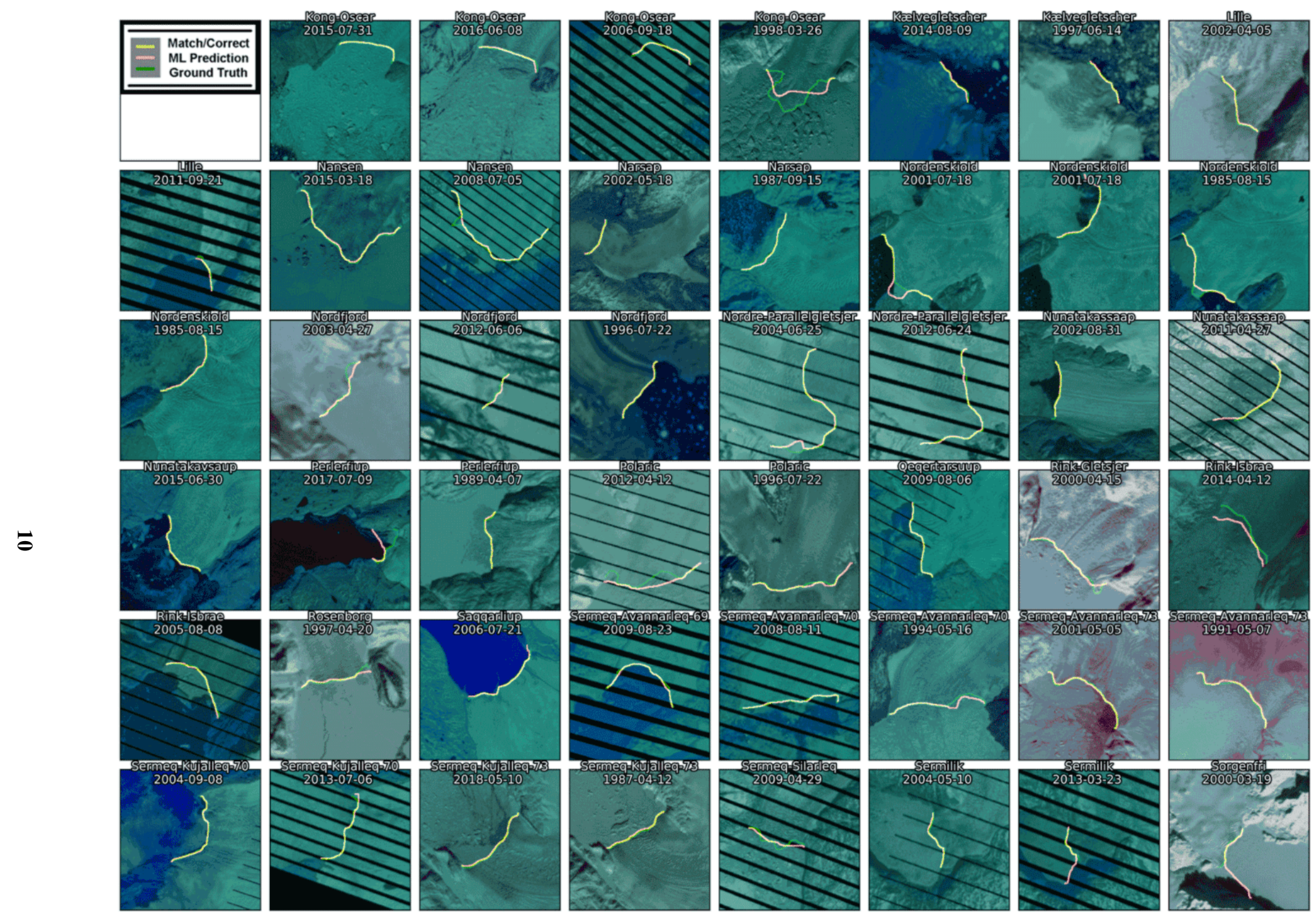

Figure S7. CALFIN validation outputs, part 3. 


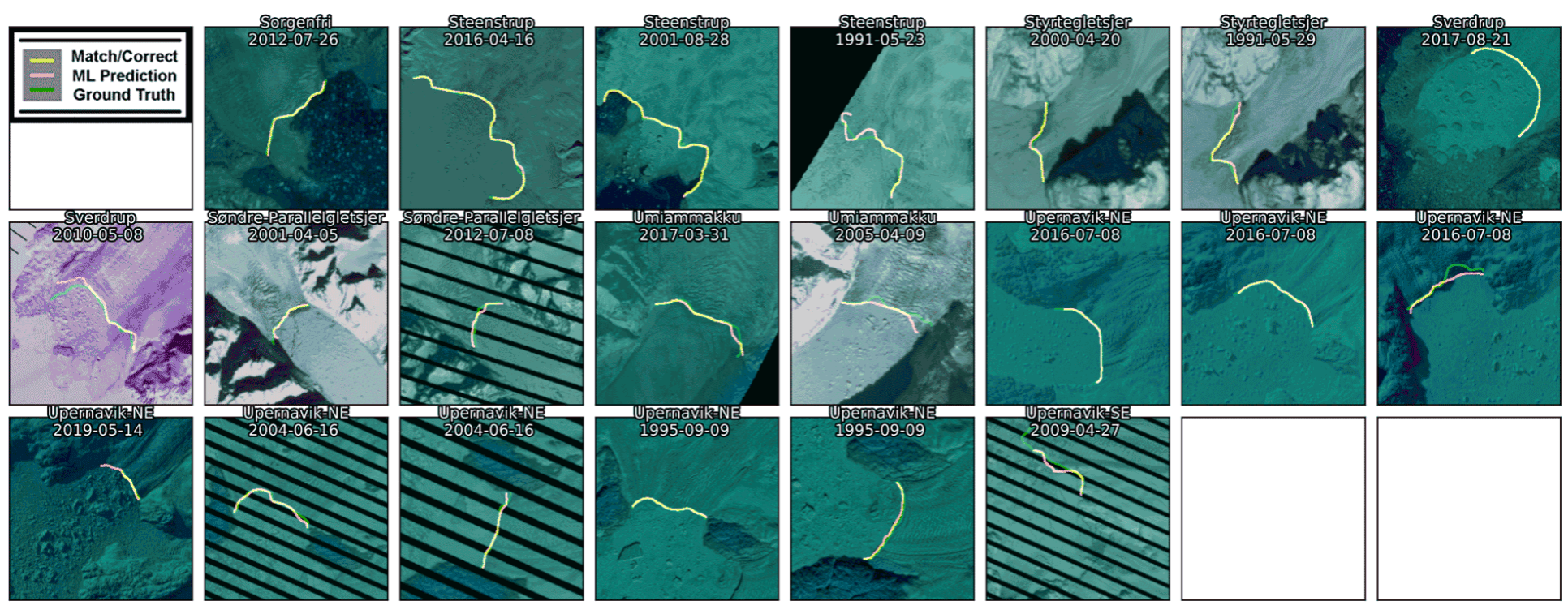

Figure S8. CALFIN validation outputs, part 4.

$\Xi$

\subsection{Mohajerani et al. Validation Set}
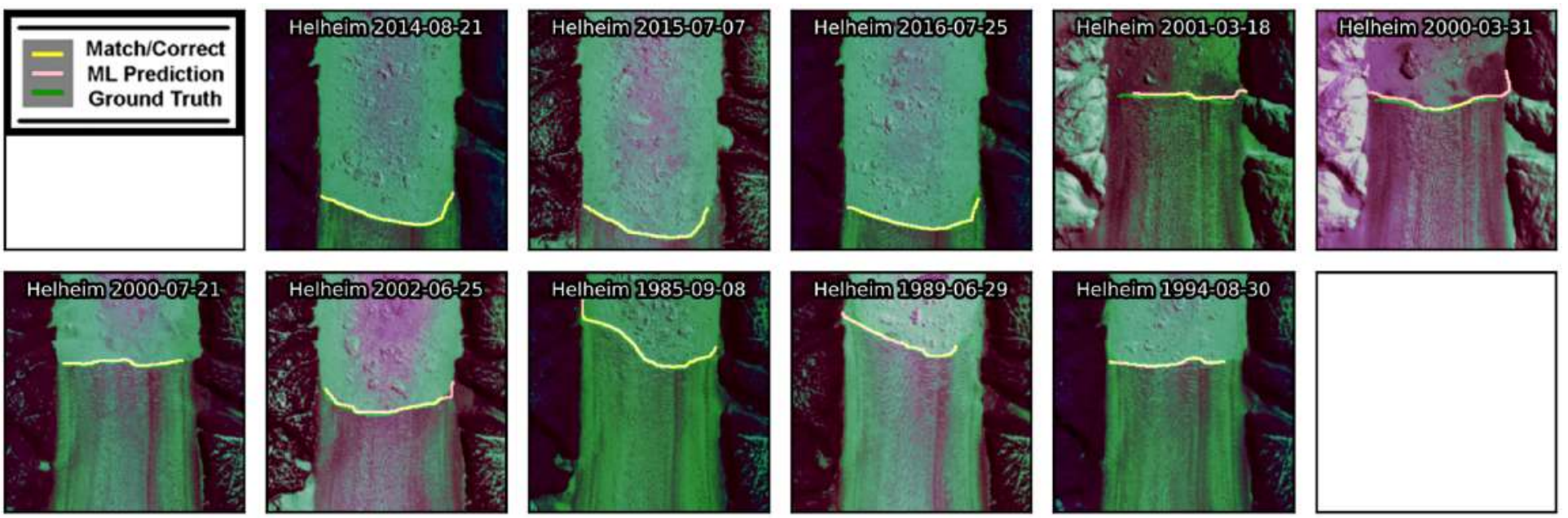

Figure S9. Mohajerani et al. validation outputs. 

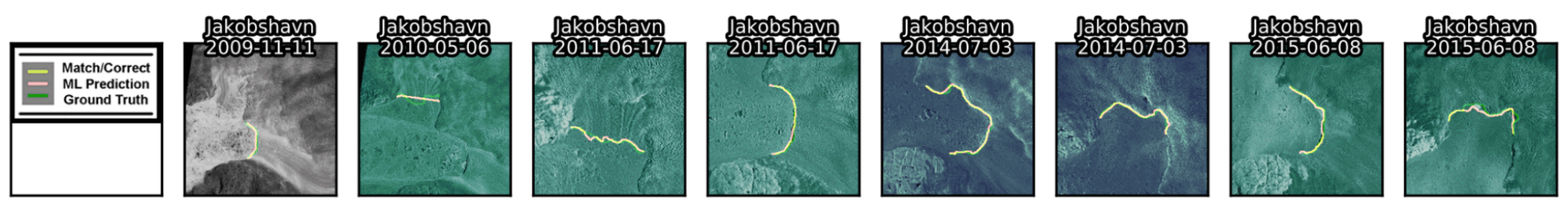

Figure S10. Zhang et al. validation outputs. Note that there are missing subsets where CALFIN did not detect any fronts, despite them being visible.

\subsection{Baumhoer et al. Validation Set}
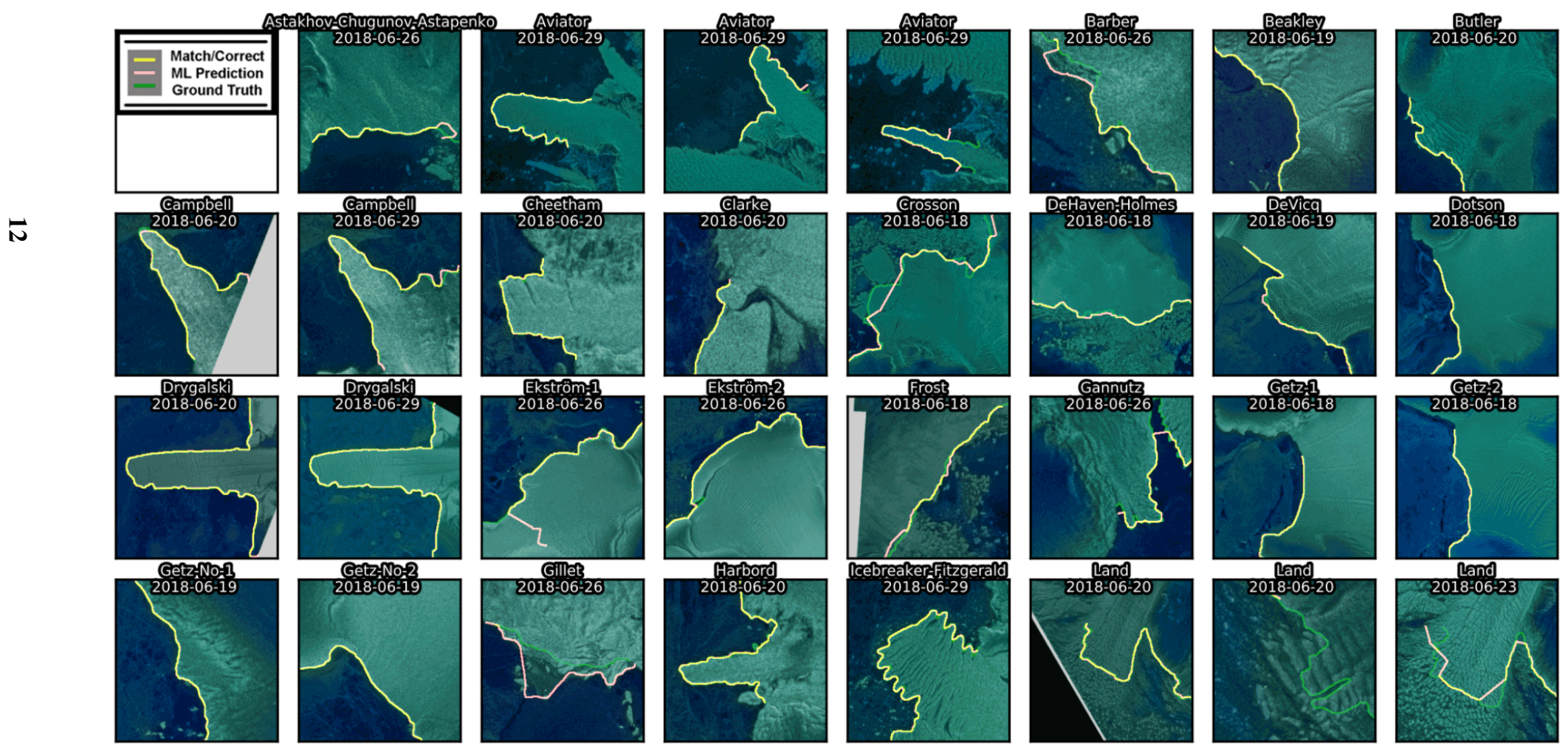

Figure S11. Baumhoer et al. validation outputs, part 1. Large errors originate from Ekstrom-1, Gillet, Land, and other similar domains. 


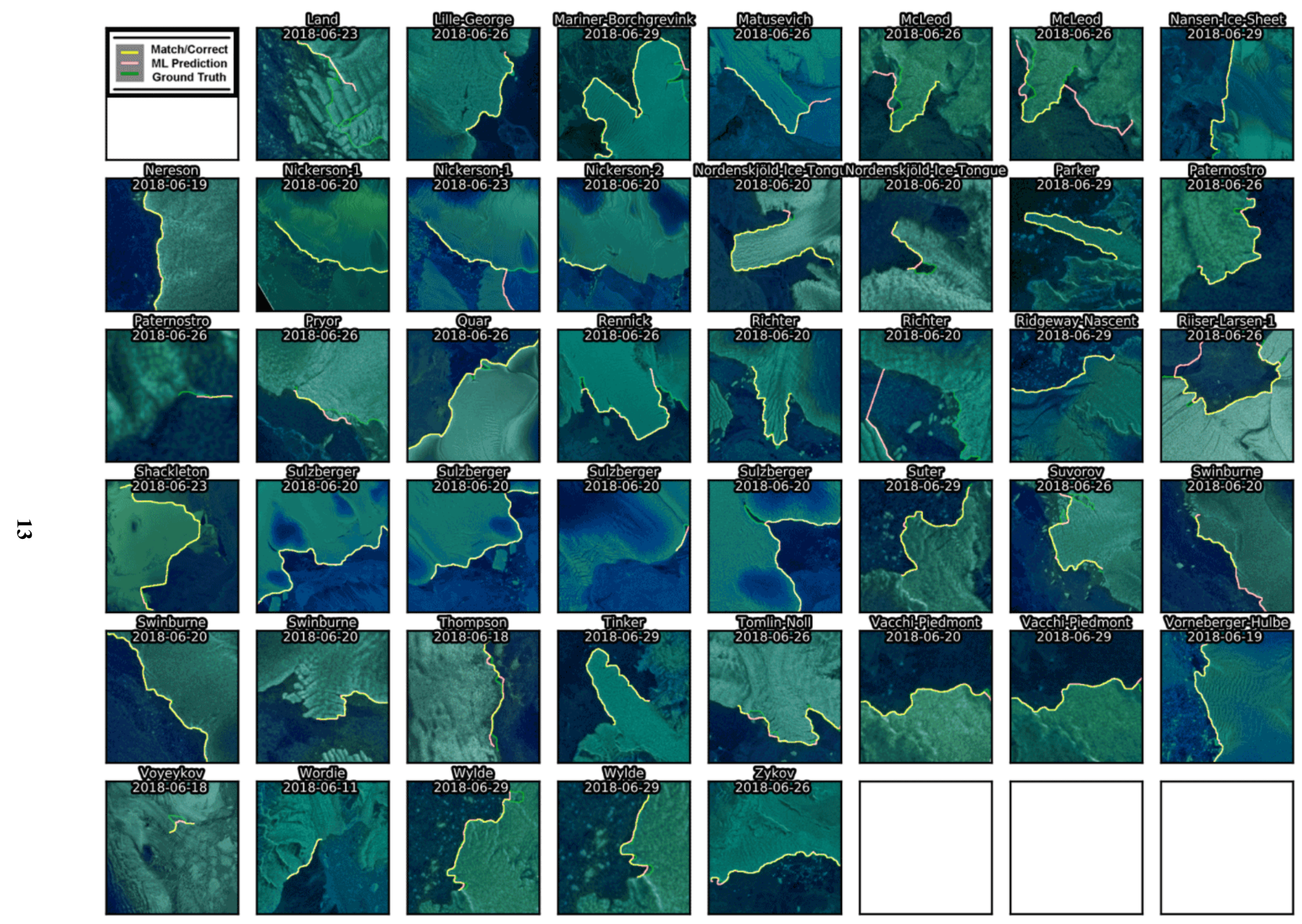

Figure S12. Baumhoer et al. validation outputs, part 2. Note that Wordie and Voyeykov, among others, have not been fully nor confidently detected. 


\section{CALFIN Validation Set Scene ID List}

Table S3: CALFIN Validation Set Scene ID List. A list of all 162 images used in the CALFIN-VS.

\begin{tabular}{|c|c|c|c|}
\hline Domain & Scene ID & Domain & Scene ID \\
\hline Akullikassaap & LE07_L1TP_014009_20000317_20170212_01_T1 & Akullikassaap & LE07_L1TP_017008_20090619_20161221_01_T1 \\
\hline Alangorssup & LC08_L1TP_016008_20150418_20170409_01_T1 & Alangorssup & LE07_L1TP_017008_20090619_20161221_01_T1 \\
\hline Alanngorliup & LE07_L1TP_009011_20010707_20170204_01_T1 & Alanngorliup & LE07_L1TP_010011_20130715_20161123_01_T1 \\
\hline Brückner & LT05_L1TP_231014_19930610_20180228_01_T2 & Cornell & LC08_L1TP_019007_20130722_20170503_01_T1 \\
\hline Cornell & LE07_L1TP_019007_20020411_20170131_01_T1 & Cornell & LT05_L1TP_019007_19900418_20170130_01_T1 \\
\hline Courtauld & LE07_L1TP_231012_20060724_20170109_01_T1 & Courtauld & LT05_L1TP_231012_19870813_20170211_01_T1 \\
\hline Dietrichson & LC08_L1TP_023006_20160811_20170322_01_T1 & Dietrichson & LE07_L1TP_023006_20080423_20161229_01_T1 \\
\hline Docker-Smith & LC08_L1TP_025006_20170422_20170501_01_T1 & Eqip & LC08_L1TP_010011_20180907_20180912_01_T1 \\
\hline Eqip & LE07_L1TP_009011_20100529_20161215_01_T1 & Fenris & LE07_L1TP_232013_20120901_20161128_01_T1 \\
\hline Frederiksborg & LE07_L1TP_229012_20040906_20170119_01_T1 & Frederiksborg & LT05_L1TP_230012_19860819_20170216_01_T1 \\
\hline Gade & LE07_L1TP_025006_20090611_20161219_01_T1 & Hayes & LC08_L1TP_018007_20150416_20170409_01_T1 \\
\hline Hayes & LC08_L1TP_080237_20160607_20170324_01_T1 & Hayes & LE07_L1TP_019007_20060406_20170110_01_T1 \\
\hline Hayes & LE07_L1TP_018007_20080810_20161225_01_T1 & Heim & LC08_L1TP_232014_20190625_20190625_01_RT \\
\hline Helheim & LC08_L1TP_233013_20130412_20170505_01_T1 & Helheim & LC08_L1TP_232014_20140729_20170420_01_T1 \\
\hline Helheim & LC08_L1TP_233013_20151011_20170403_01_T1 & Helheim & LC08_L1TP_233013_20160911_20170321_01_T1 \\
\hline Helheim & LC08_L1TP_232013_20180606_20180615_01_T1 & Helheim & LE07_L1TP_232014_20000527_20170211_01_T1 \\
\hline Helheim & LE07_L1TP_232013_20020517_20170130_01_T1 & Helheim & LE07_L1TP_232013_20030808_20170124_01_T1 \\
\hline Helheim & LE07_L1TP_232013_20060512_20170109_01_T2 & Helheim & LE07_L1TP_232013_20100405_20161215_01_T2 \\
\hline Helheim & LE07_L1TP_232013_20110627_20161208_01_T1 & Helheim & LE07_L1TP_232013_20130803_20161123_01_T1 \\
\hline Helheim & LT05_L1TP_232014_19860817_20170216_01_T1 & Helheim & LT05_L1TP_232013_19880907_20170206_01_T1 \\
\hline Helheim & LT05_L1TP_233013_19910603_20180228_01_T2 & Helheim & LT05_L1TP_232013_19910714_20180228_01_T1 \\
\hline Helheim & LT05_L1TP_232013_19920529_20180228_01_T2 & Helheim & LT05_L1TP_232013_19920614_20180228_01_T2 \\
\hline Helheim & LT05_L1TP_232013_19920902_20180228_01_T1 & Helheim & LT05_L1TP_232014_19930430_20180228_01_T2 \\
\hline Helheim & LT05_L1TP_233013_19930608_20180228_01_T2 & Helheim & LT05_L1TP_233013_19940830_20170113_01_T2 \\
\hline Helheim & LT05_L1TP_232013_19950420_20180228_01_T2 & Helheim & LT05_L1TP_232014_19950420_20180228_01_T2 \\
\hline Helheim & LT05_L1TP_232014_19950826_20180228_01_T1 & Helheim & LT05_L1TP_233013_19960702_20180228_01_T1 \\
\hline Helheim & LT05_L1TP_232013_19970511_20180228_01_T2 & Helheim & LT05_L1TP_232013_19970628_20180228_01_T1 \\
\hline
\end{tabular}

Continued on next page 
Table S3 - Continued from previous page

\begin{tabular}{|c|c|c|c|}
\hline Domain & Scene ID & Domain & Scene ID \\
\hline Helheim & LT05_L1TP_232013_19980530_20161224_01_T1 & Helheim & LT05_L1TP_232013_19980903_20161222_01_T1 \\
\hline Hutchinson & LE07_L1TP_230012_20050831_20170114_01_T1 & Hutchinson & LE07_L1TP_230012_20130501_20161124_01_T2 \\
\hline Illullip & LE07_L1TP_021007_20010422_20170206_01_T1 & Illullip & LE07_L1TP_019007_20130527_20161124_01_T1 \\
\hline Inngia & LC08_L1TP_014009_20180615_20180703_01_T1 & Inngia & LE07_L1TP_013009_20050425_20170115_01_T1 \\
\hline Inngia & LE07_L1TP_014009_20070406_20170104_01_T1 & Inngia & LT05_L1TP_013009_19850917_20170218_01_T1 \\
\hline Inngia & LT05_L1TP_013009_19930416_20180220_01_T1 & Issuusarsuit & LE07_L1TP_024006_20050812_20170113_01_T1 \\
\hline Jakobshavn & LC08_L1TP_008012_20131029_20170429_01_T1 & Jakobshavn & LC08_L1TP_008012_20170602_20170615_01_T1 \\
\hline Jakobshavn & LE07_L1TP_010011_20010324_20170206_01_T1 & Jakobshavn & LT05_L1TP_008011_19860512_20170218_01_T1 \\
\hline Jakobshavn & LT05_L1TP_008011_19950505_20180220_01_T1 & Kakiffaat & LE07_L1TP_017008_20050827_20170114_01_T1 \\
\hline Kangerdluarssup & LC08_L1TP_013009_20170723_20170809_01_T1 & Kangerdluarssup & LE07_L1TP_011010_20130519_20161124_01_T1 \\
\hline Kangerlussuaq & LE07_L1TP_231012_20030427_20170125_01_T2 & Kangerlussuaq & LE07_L1TP_231012_20120419_20161202_01_T2 \\
\hline Kangerlussuaq & LT05_L1TP_229012_19970607_20180227_01_T1 & Kangerlussuup & LE07_L1TP_012010_20030920_20170124_01_T1 \\
\hline Kangerlussuup & LE07_L1TP_014009_20090902_20161220_01_T1 & Kangerlussuup & LT05_L1TP_011010_20090617_20161025_01_T1 \\
\hline Kangiata-Nunaata & LC08_L1TP_005015_20170613_20170628_01_T1 & Kangiata-Nunaata & LE07_L1TP_005015_20020815_20170128_01_T1 \\
\hline Kangiata-Nunaata & LT05_L1TP_006015_19950405_20170109_01_T1 & Kangilleq & LE07_L1TP_011010_20060703_20170109_01_T1 \\
\hline Kjer & LC08_L1TP_019007_20150914_20170404_01_T1 & Kjer & LE07_L1TP_019007_20020411_20170131_01_T1 \\
\hline Kjer & LT05_L1TP_019007_19870613_20170212_01_T1 & Kong-Oscar & LC08_L1TP_024006_20150731_20170406_01_T1 \\
\hline Kong-Oscar & LC08_L1TP_023006_20160608_20170324_01_T1 & Kong-Oscar & LE07_L1TP_022006_20060918_20170107_01_T1 \\
\hline Kong-Oscar & LT05_L1TP_024006_19980326_20161225_01_T1 & Kælvegletscher & LC08_L1TP_229012_20140809_20170420_01_T1 \\
\hline Kælvegletscher & LE07_L1TP_231012_20050603_20170114_01_T2 & Kælvegletscher & LE07_L1TP_231012_20120825_20161129_01_T1 \\
\hline Kælvegletscher & LT05_L1TP_230012_19970614_20180227_01_T1 & Lille & LE07_L1TP_009011_20020405_20170131_01_T1 \\
\hline Lille & LE07_L1TP_009011_20110921_20161206_01_T1 & Midgård & LT05_L1TP_232013_19950927_20180228_01_T1 \\
\hline Nansen & LC08_L1TP_023006_20150318_20170412_01_T1 & Nansen & LE07_L1TP_022006_20080705_20161228_01_T1 \\
\hline Narsap & LE07_L1TP_006015_20020518_20170130_01_T1 & Narsap & LT05_L1TP_005015_19870915_20170211_01_T1 \\
\hline Nordenskiold & LE07_L1TP_022006_20010718_20170204_01_T1 & Nordenskiold & LT05_L1TP_022006_19850815_20170219_01_T1 \\
\hline Nordfjord & LE07_L1TP_231012_20030427_20170125_01_T2 & Nordfjord & LE07_L1TP_231012_20120606_20161202_01_T1 \\
\hline Nordfjord & LT05_L1TP_229012_19960722_20180227_01_T1 & Nordre-Parallelgletsjer & LE07_L1TP_230012_20040625_20170120_01_T1 \\
\hline Nordre-Parallelgletsjer & LE07_L1TP_229012_20120624_20161130_01_T1 & Nordre-Parallelgletsjer & LT05_L1TP_229012_19970522_20180227_01_T2 \\
\hline Nunatakassaap & LE07_L1TP_021007_20020831_20170128_01_T1 & Nunatakassaap & LE07_L1TP_020007_20110427_20161209_01_T1 \\
\hline Nunatakavsaup & LC08_L1TP_015008_20150630_20170407_01_T1 & Nunatakavsaup & LE07_L1TP_016008_20070522_20170103_01_T1 \\
\hline
\end{tabular}


Table S3 - Continued from previous page

\begin{tabular}{|c|c|c|c|}
\hline Domain & Scene ID & Domain & Scene ID \\
\hline Perlerfiup & LC08_L1TP_011010_20170709_20170717_01_T1 & Perlerfiup & LT05_L1TP_011010_19890407_20170204_01_T1 \\
\hline Polaric & LE07_L1TP_230012_20120412_20161202_01_T2 & Polaric & LT05_L1TP_229012_19960722_20180227_01_T1 \\
\hline Qeqertarsuup & LE07_L1TP_017008_20090806_20161218_01_T1 & Rink-Gletsjer & LE07_L1TP_025006_20000415_20170212_01_T1 \\
\hline Rink-Isbrae & LC08_L1TP_011010_20140412_20170423_01_T1 & Rink-Isbrae & LE07_L1TP_012010_20050808_20170114_01_T1 \\
\hline Rink-Isbrae & LT05_L1TP_013009_19860616_20170217_01_T1 & Rink-Isbrae & LT05_L1TP_012010_19900519_20170130_01_T1 \\
\hline Rosenborg & LT05_L1TP_229012_19970420_20180227_01_T2 & Saqqarliup & LE07_L1TP_009011_20060721_20170109_01_T1 \\
\hline Sermeq-Avannarleq-69 & LC08_L1TP_008012_20181011_20181030_01_T1 & Sermeq-Avannarleq-69 & LE07_L1TP_008011_20090823_20161218_01_T1 \\
\hline Sermeq-Avannarleq-70 & LC08_L1TP_011010_20140428_20170423_01_T1 & Sermeq-Avannarleq-70 & LE07_L1TP_009011_20080811_20161225_01_T1 \\
\hline Sermeq-Avannarleq-70 & LT05_L1TP_010011_19940516_20170115_01_T1 & Sermeq-Avannarleq-73 & LE07_L1TP_016008_20010505_20170205_01_T1 \\
\hline Sermeq-Avannarleq-73 & LT05_L1TP_019007_19910507_20180221_01_T2 & Sermeq-Kujalleq-70 & LE07_L1TP_010011_20040908_20170119_01_T1 \\
\hline Sermeq-Kujalleq-70 & LE07_L1TP_011010_20130706_20161123_01_T1 & Sermeq-Kujalleq-73 & LC08_L1TP_018008_20180510_20180517_01_T1 \\
\hline Sermeq-Kujalleq-73 & LT05_L1TP_017008_19870412_20170213_01_T1 & Sermeq-Silarleq & LE07_L1TP_012010_20090429_20161220_01_T1 \\
\hline Sermilik & LE07_L1TP_011010_20040510_20170120_01_T1 & Sermilik & LE07_L1TP_012010_20130323_20161124_01_T1 \\
\hline Sorgenfri & LE07_L1TP_229012_20000319_20170212_01_T2 & Sorgenfri & LE07_L1TP_229012_20120726_20161130_01_T1 \\
\hline Steenstrup & LC08_L1TP_020007_20160416_20170326_01_T1 & Steenstrup & LE07_L1TP_021007_20010828_20170203_01_T1 \\
\hline Steenstrup & LT05_L1TP_019007_19910523_20180221_01_T1 & Styrtegletsjer & LE07_L1TP_229012_20000420_20170212_01_T2 \\
\hline Styrtegletsjer & LE07_L1TP_229012_20100416_20161214_01_T1 & Styrtegletsjer & LT05_L1TP_230012_19910529_20180227_01_T1 \\
\hline Sverdrup & LC08_L1TP_024006_20170821_20170911_01_T1 & Sverdrup & LE07_L1TP_022006_20100508_20161214_01_T1 \\
\hline Søndre-Parallelgletsjer & LE07_L1TP_231012_20010405_20170205_01_T2 & Søndre-Parallelgletsjer & LE07_L1TP_231012_20120708_20161130_01_T1 \\
\hline Umiammakku & LC08_L1TP_015009_20170331_20170414_01_T1 & Umiammakku & LE07_L1TP_013009_20050409_20170115_01_T1 \\
\hline Umiammakku & LE07_L1TP_014009_20110620_20161209_01_T1 & Upernavik-NE & LC08_L1TP_015008_20130928_20170502_01_T1 \\
\hline Upernavik-NE & LC08_L1TP_082235_20160708_20170323_01_T1 & Upernavik-NE & LC08_L1TP_082236_20190514_20190521_01_T1 \\
\hline Upernavik-NE & LE07_L1TP_014009_20040616_20170120_01_T1 & Upernavik-NE & LT05_L1TP_017008_19950909_20180220_01_T1 \\
\hline Upernavik-SE & LC08_L1TP_016008_20160506_20170326_01_T1 & Upernavik-SE & LE07_L1TP_014009_20090427_20161222_01_T1 \\
\hline
\end{tabular}


8 Selected Relative Advance and Retreat Graphs
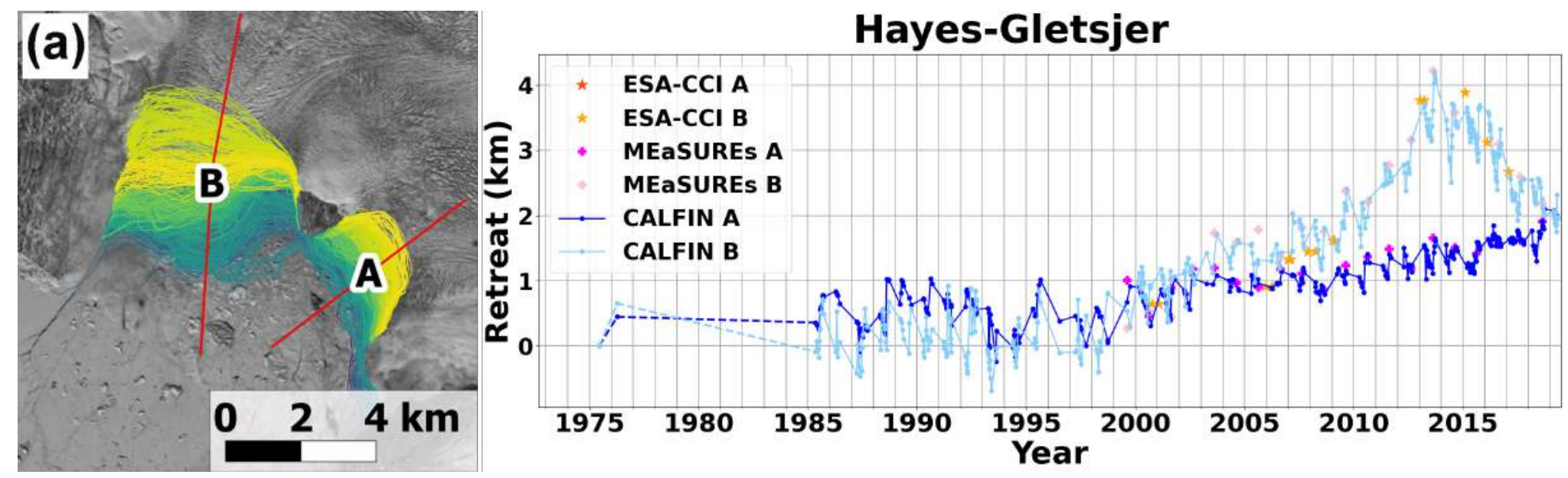

Figure S13. Terminus Advance and Retreat Over Time for Hayes Gletsjer. Dotted lines from 1972-1985 that indicate a lack of seasonal observations.

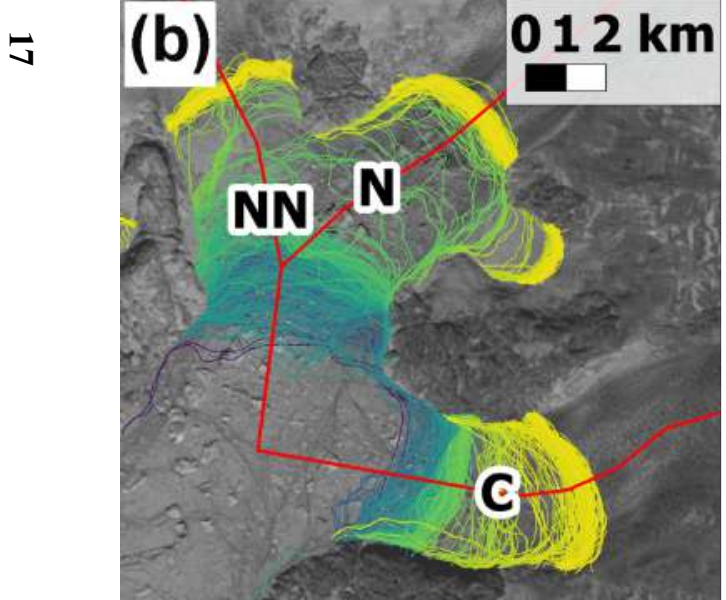

\section{Upernavik-Isstrom-N-C}

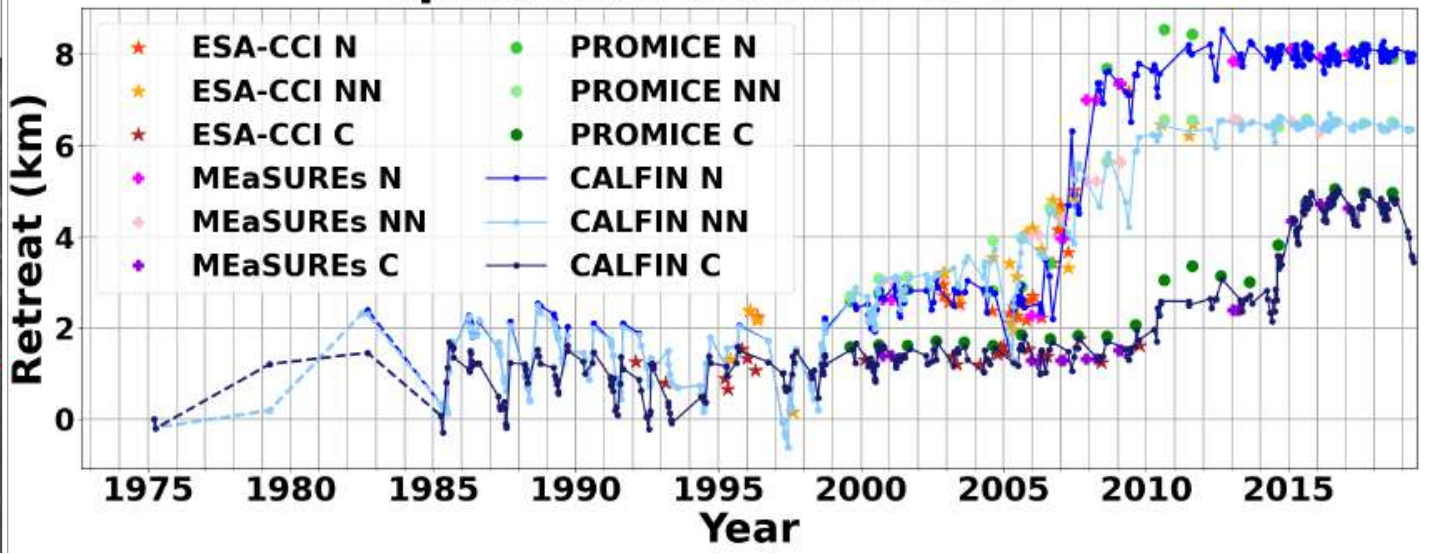

Figure S14. Terminus Advance and Retreat Over Time for Upernavik Isstrom N-C. Dotted lines from 1972-1985 that indicate a lack of seasonal observations. 


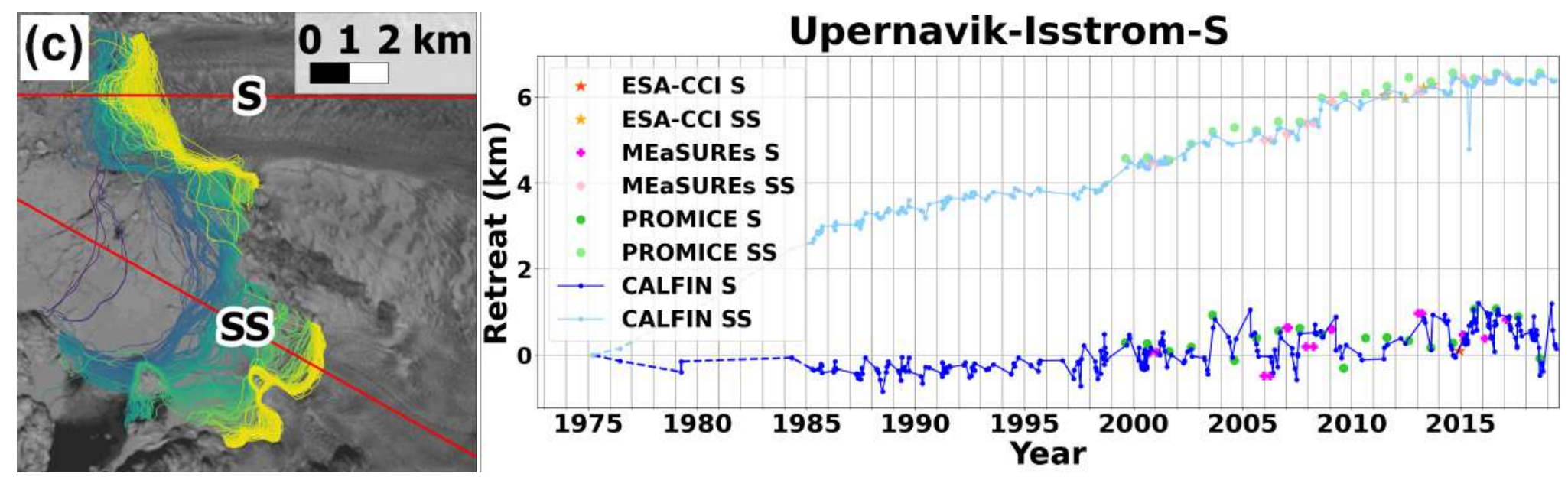

Figure S15. Terminus Advance and Retreat Over Time for Upernavik Isstrom S. Dotted lines from 1972-1985 that indicate a lack of seasonal observations.
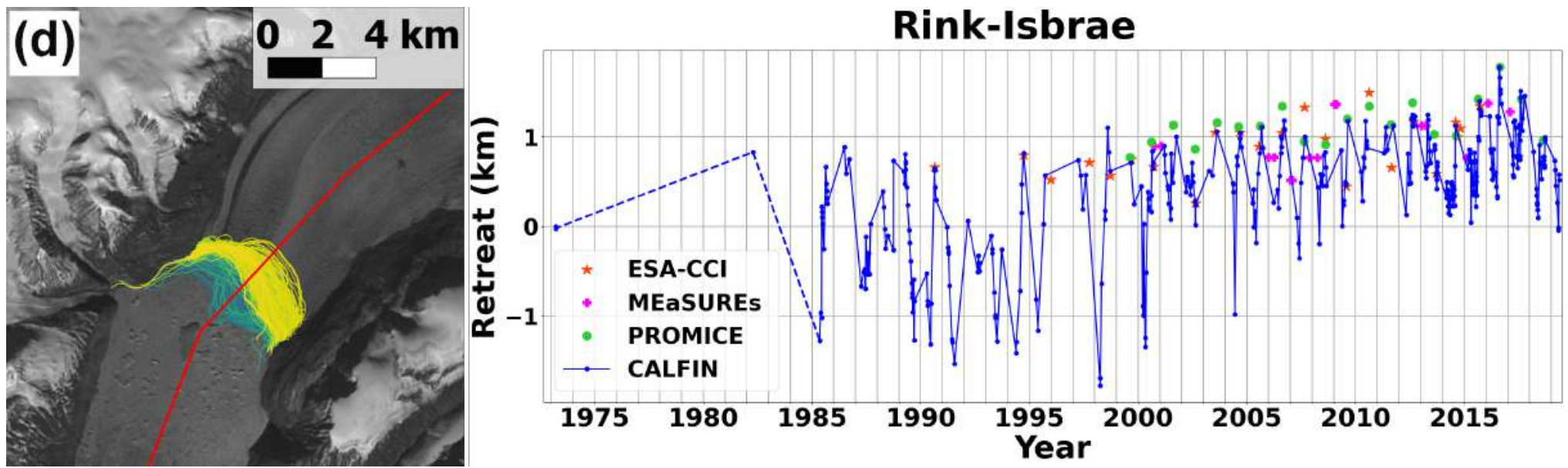

Figure S16. Terminus Advance and Retreat Over Time for Rink Isbrae. Dotted lines from 1972-1985 that indicate a lack of seasonal observations. 

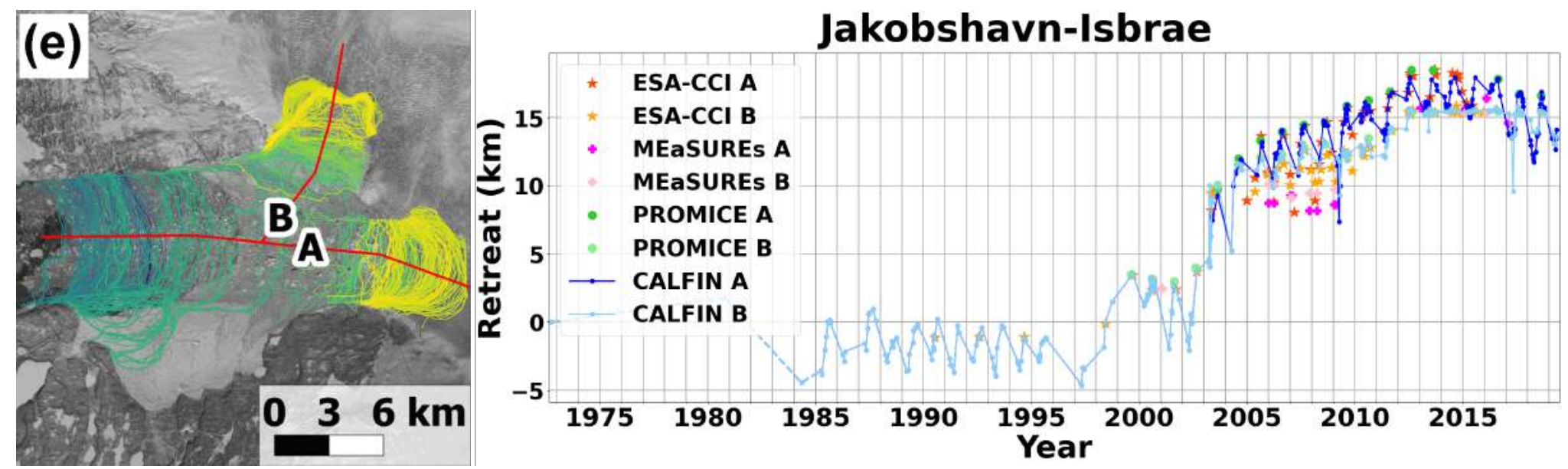

Figure S17. Terminus Advance and Retreat Over Time for Jakobshavn Isbrae. Dotted lines from 1972-1985 that indicate a lack of seasonal observations.

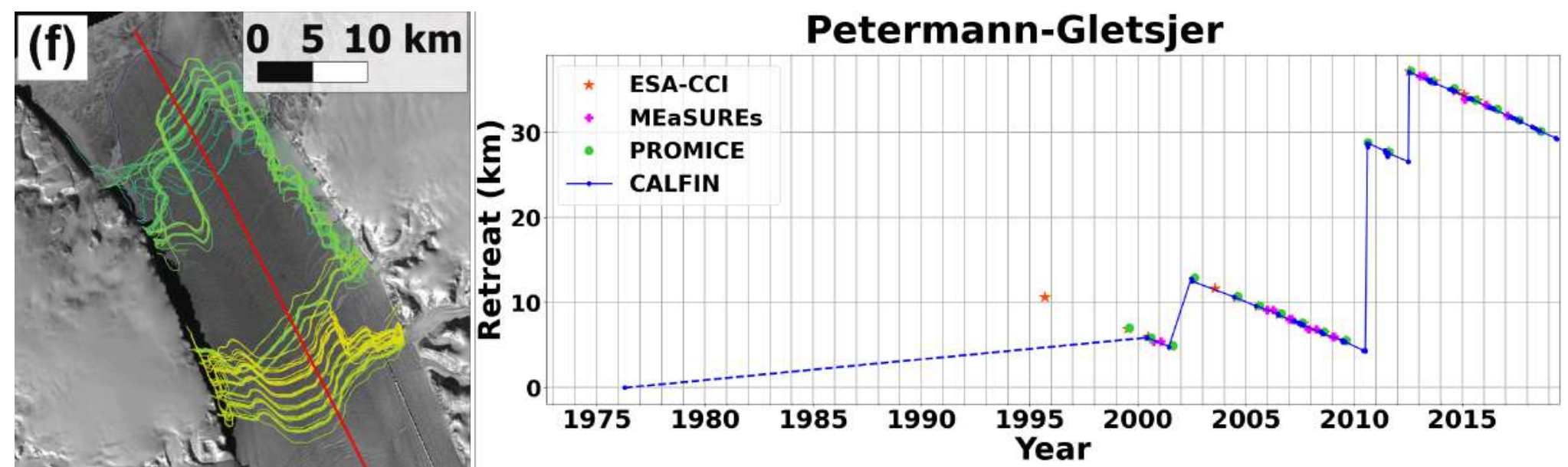

Figure S18. Terminus Advance and Retreat Over Time for Petermann Gletsjer. Dotted lines from 1972-1985 that indicate a lack of seasonal observations. 

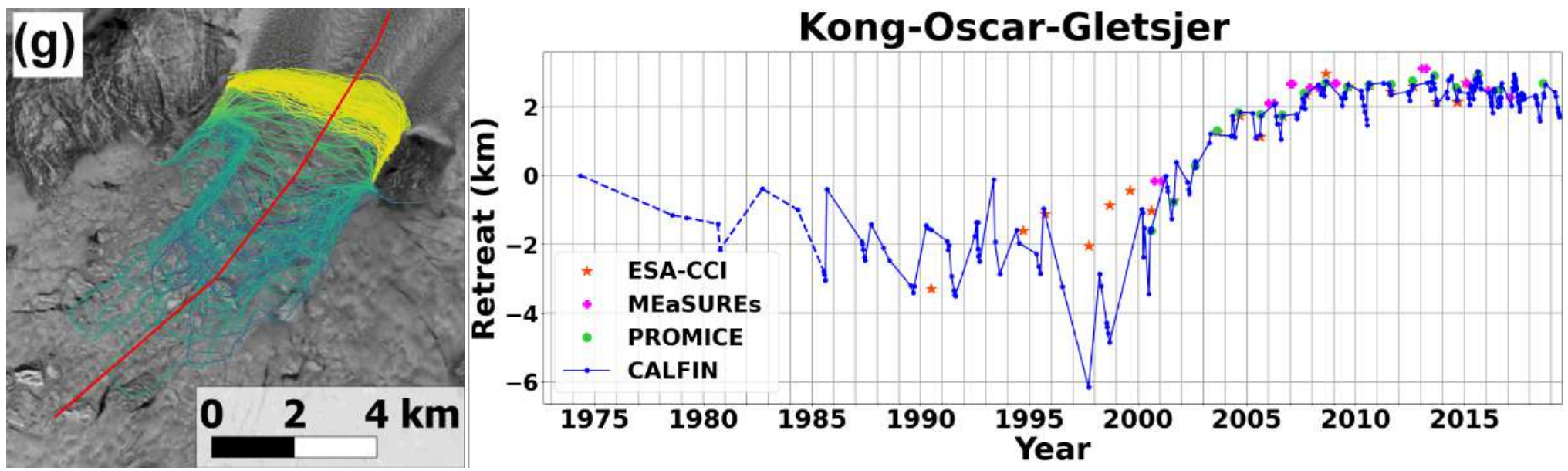

Figure S19. Terminus Advance and Retreat Over Time for Kong Oscar Gletsjer. Dotted lines from 1972-1985 that indicate a lack of seasonal observations.

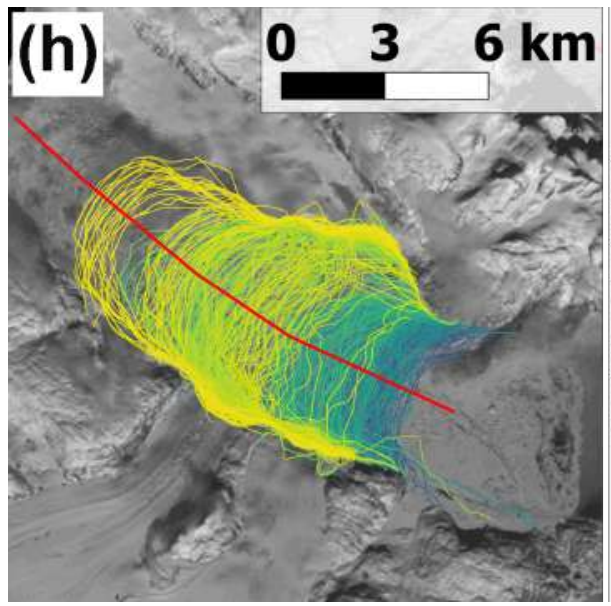

\section{Kangerlussuaq-Gletsjer}

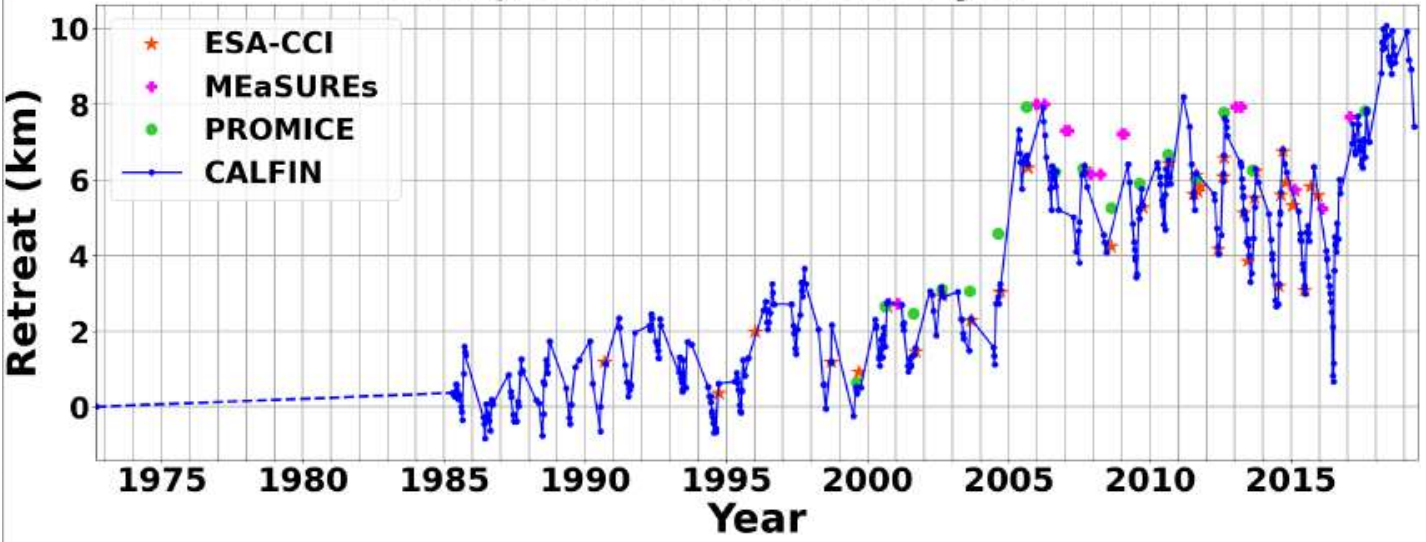

Figure S20. Terminus Advance and Retreat Over Time for Kangerlussuaq Gletsjer. Dotted lines from 1972-1985 that indicate a lack of seasonal observations. 

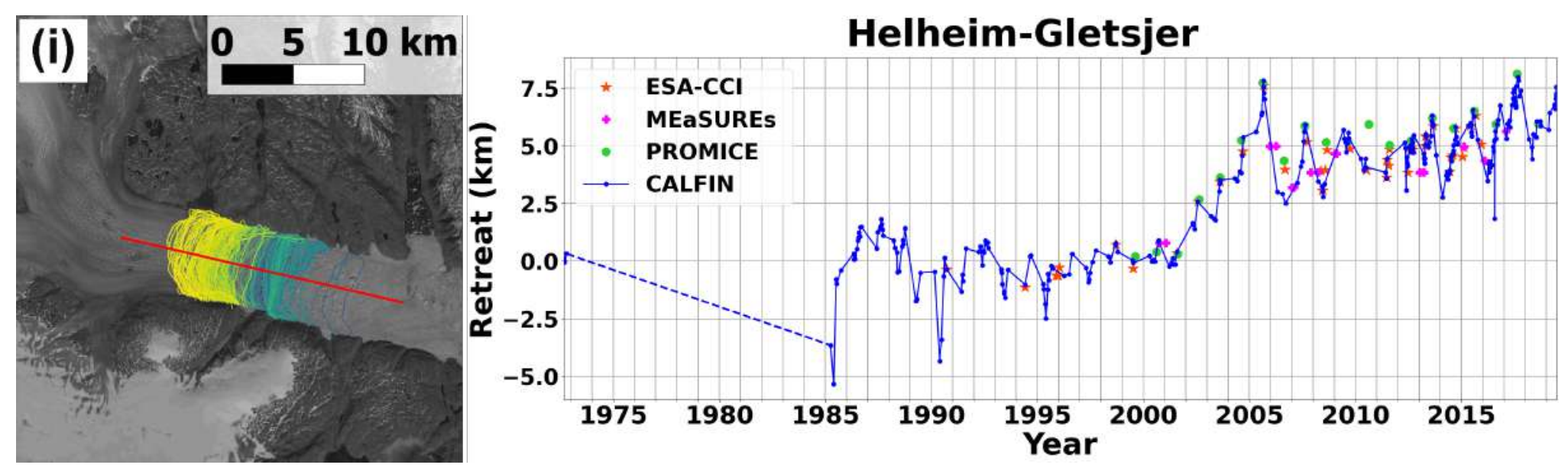

Figure S21. Terminus Advance and Retreat Over Time for Helheim Gletsjer. Dotted lines from 1972-1985 that indicate a lack of seasonal observations.
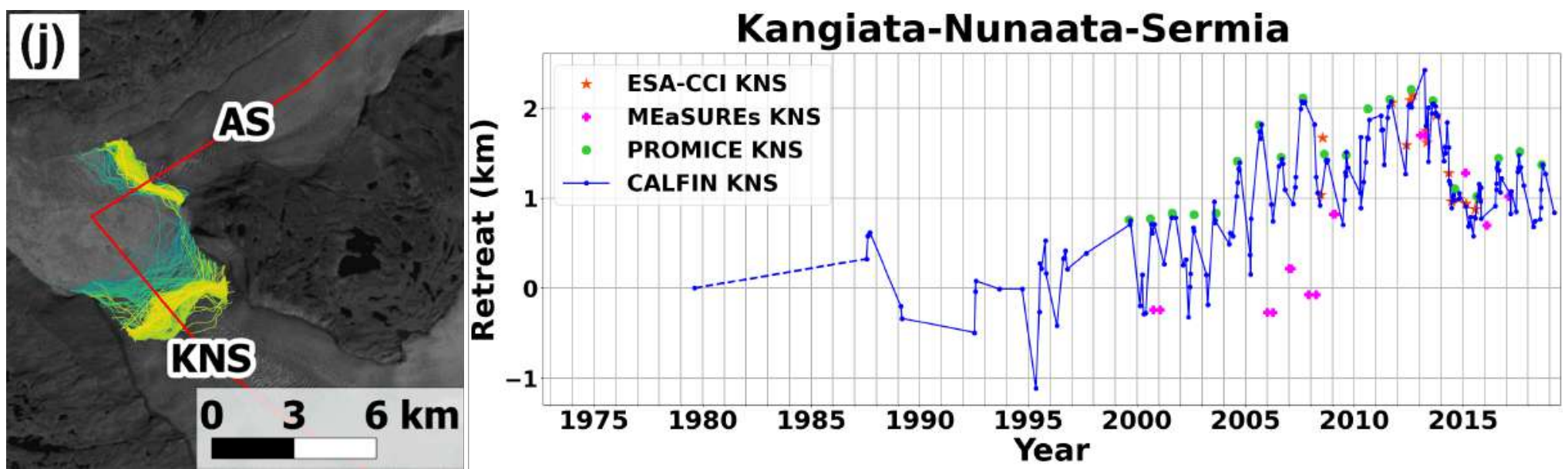

Figure S22. Terminus Advance and Retreat Over Time for Kangiata Nunaata Sermia. Dotted lines from 1972-1985 that indicate a lack of seasonal observations. 
9 Regional Relative Advance and Retreat Graphs

Greenland (87 glaciers)

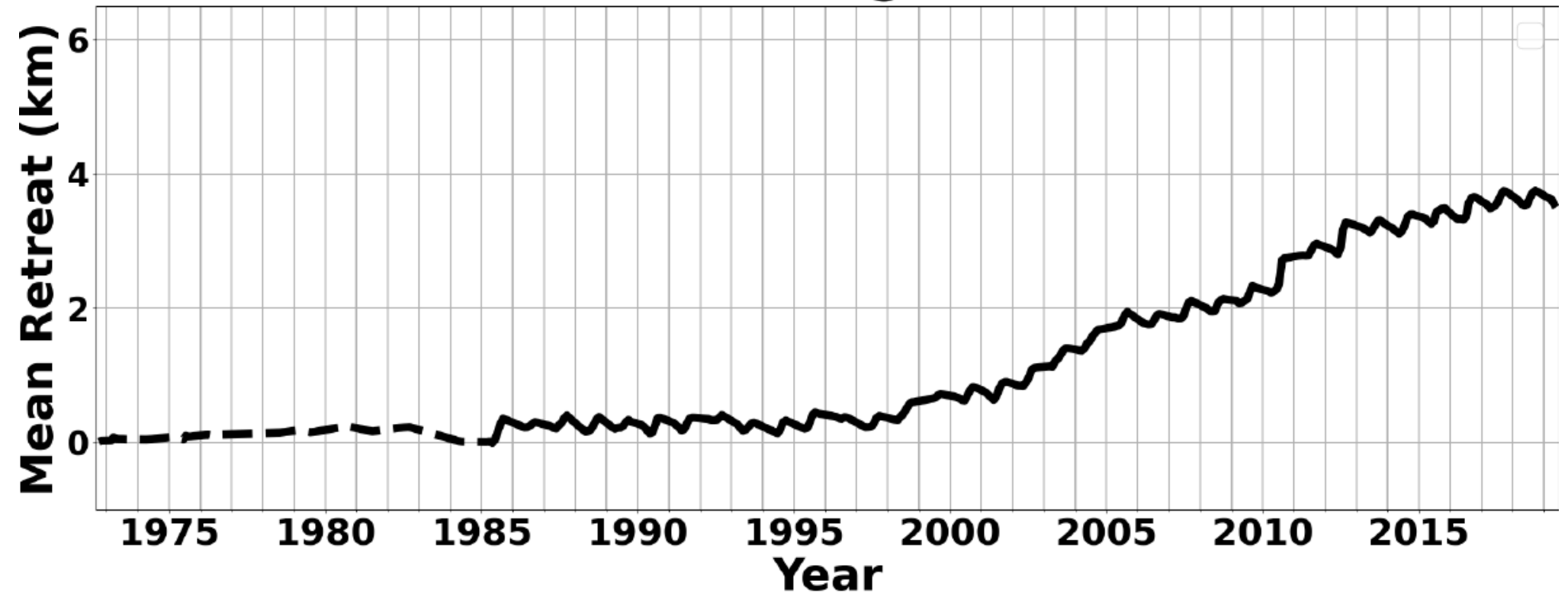

Figure S23. Regional Terminus Advance and Retreat Over Time for Greenland. Dotted lines from 1972-1985 that indicate a lack of seasonal observations. 


\section{NW Greenland (41 glaciers)}

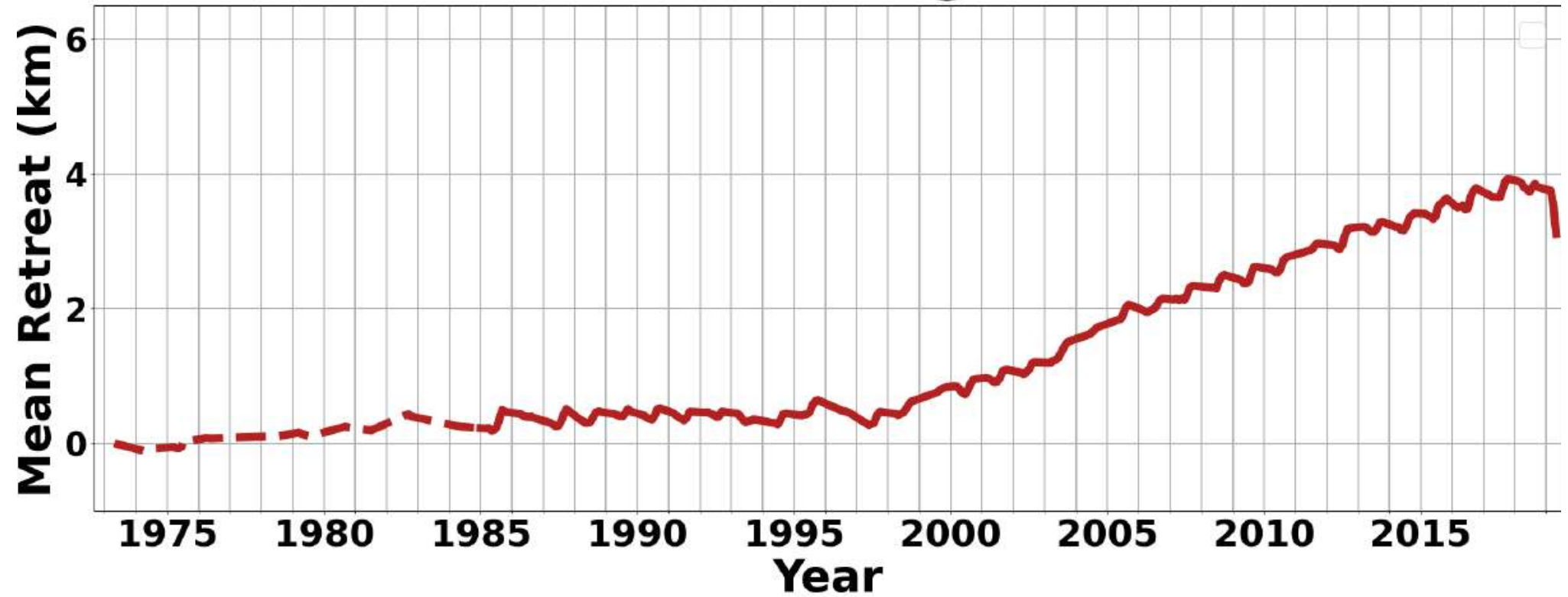

Figure S24. Regional Terminus Advance and Retreat Over Time for NW Greenland. Dotted lines from 1972-1985 that indicate a lack of seasonal observations. 


\section{CW Greenland (17 glaciers)}

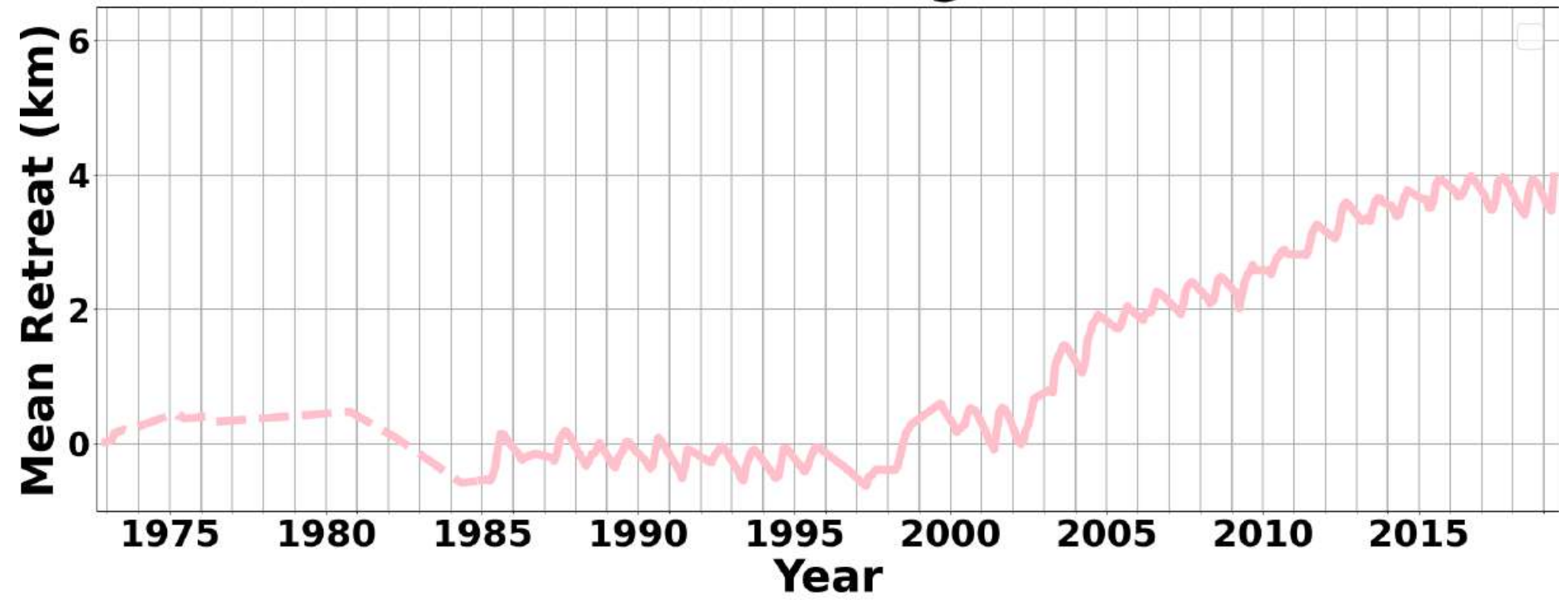

Figure S25. Regional Terminus Advance and Retreat Over Time for CW Greenland. Dotted lines from 1972-1985 that indicate a lack of seasonal observations. 


\section{CE Greenland (17 glaciers)}

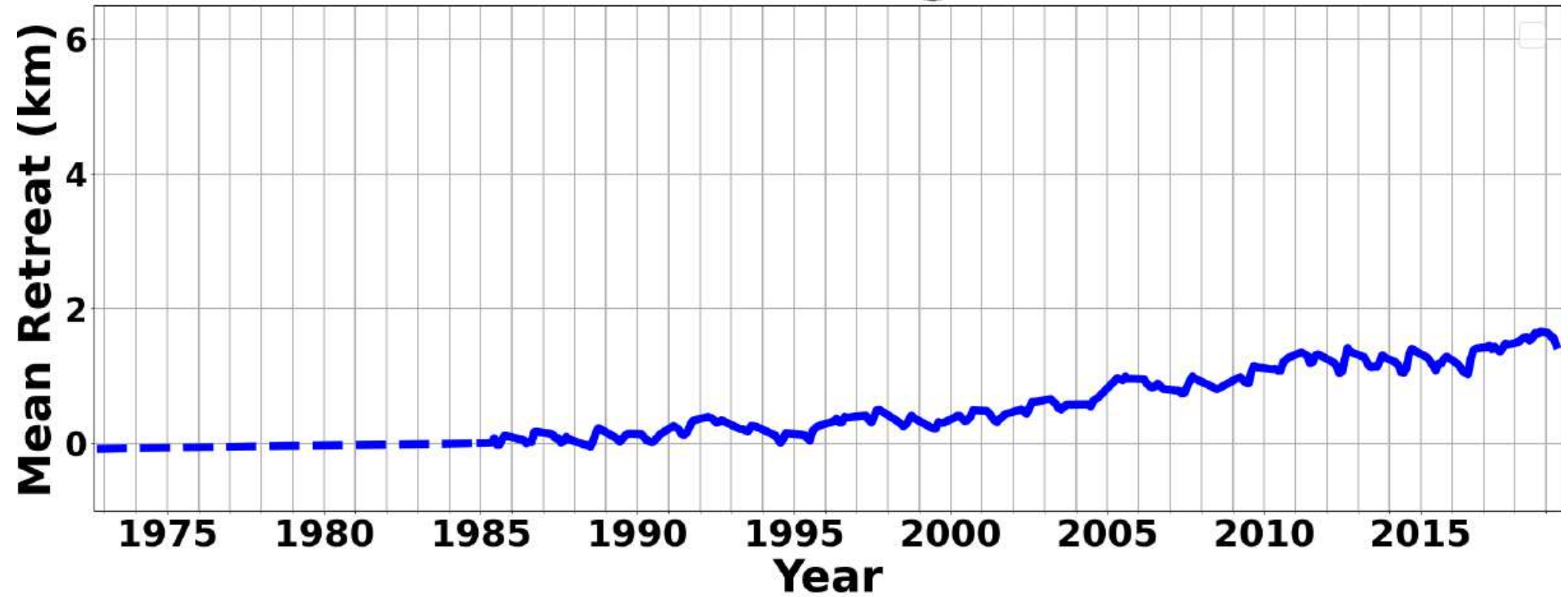

Figure S26. Regional Terminus Advance and Retreat Over Time for CE Greenland. Dotted lines from 1972-1985 that indicate a lack of seasonal observations. 


\section{SE Greenland (7 glaciers)}

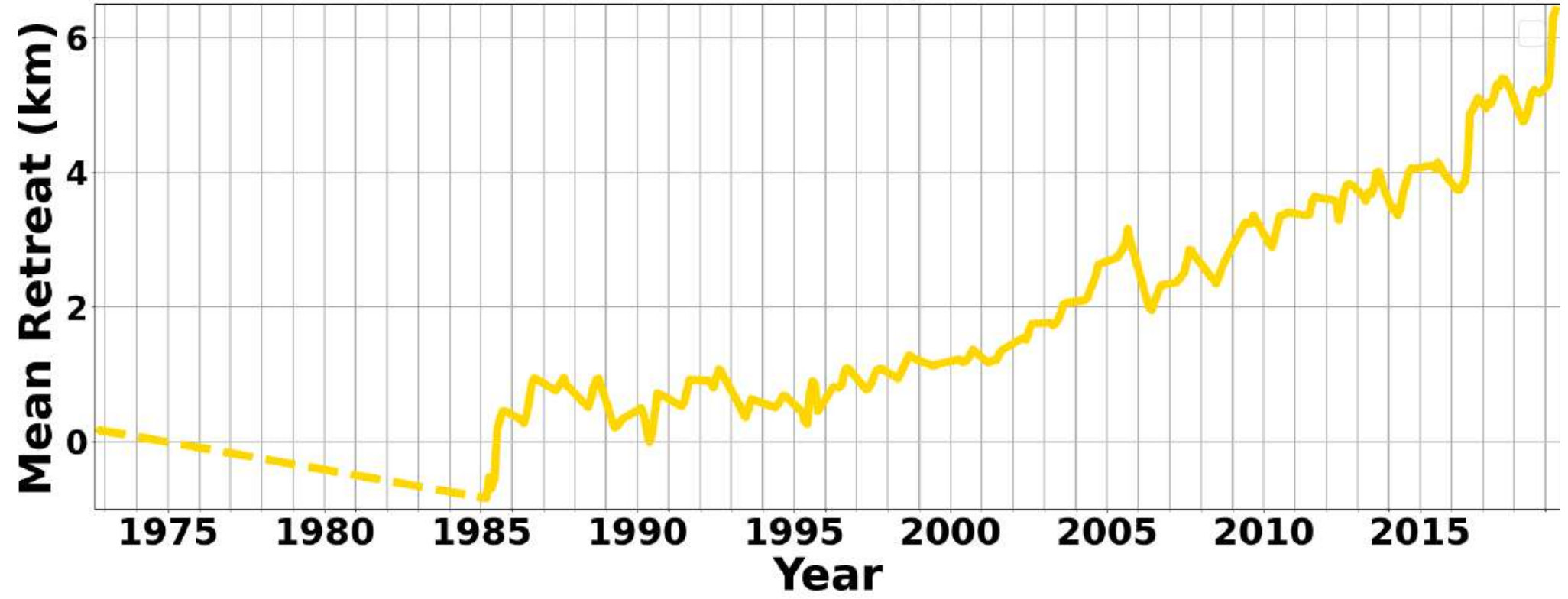

Figure S27. Regional Terminus Advance and Retreat Over Time for SE Greenland. Dotted lines from 1972-1985 that indicate a lack of seasonal observations. 


\section{SW Greenland (4 glaciers)}

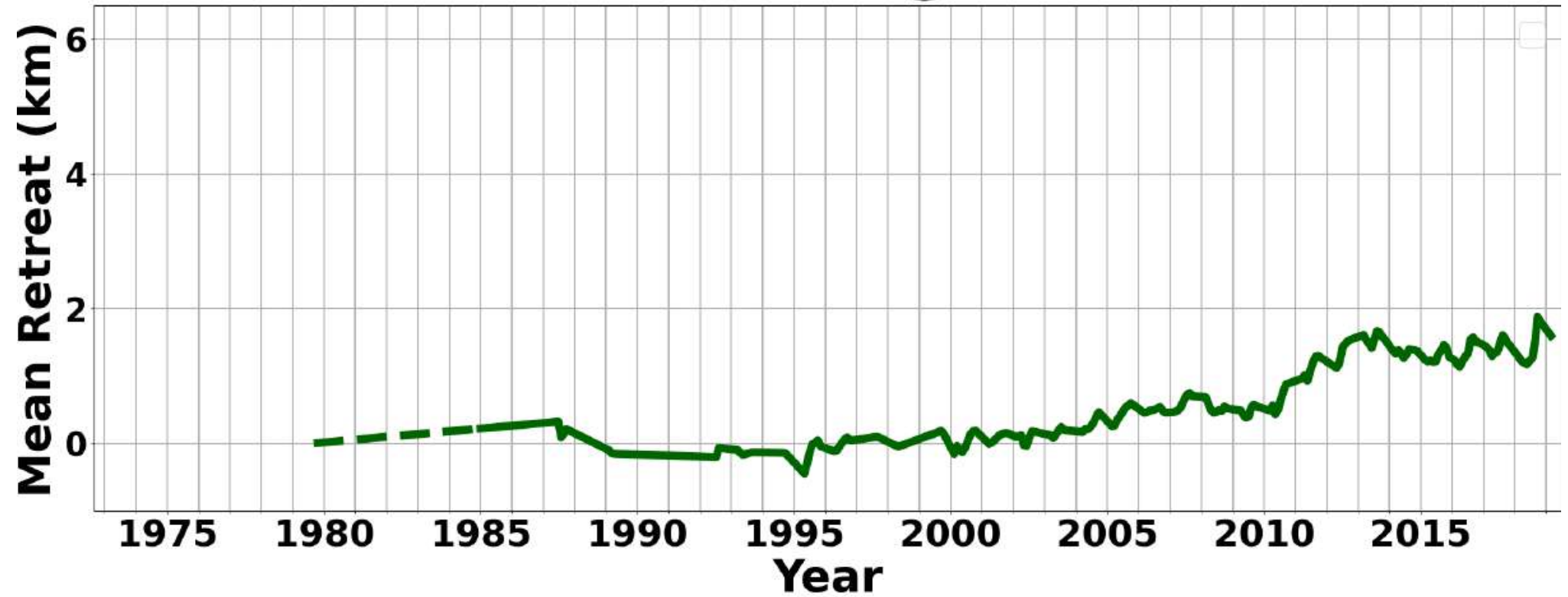

Figure S28. Regional Terminus Advance and Retreat Over Time for SW Greenland. Dotted lines from 1972-1985 that indicate a lack of seasonal observations. 